\title{
TOPOLOGICAL HOCHSCHILD HOMOLOGY AND HIGHER CHARACTERISTICS
}

\author{
JONATHAN A. CAMPBELL AND KATE PONTO
}

\begin{abstract}
We show that an important classical fixed point invariant, the Reidemeister trace, arises as a topological Hochschild homology transfer. This generalizes a corresponding classical result for the Euler characteristic and is a first step in showing the Reidemeister trace is in the image of the cyclotomic trace. The main result follows from developing the relationship between shadows [Pon10], topological Hochschild homology, and Morita invariance in bicategorical generality.
\end{abstract}

\section{CONTENTS}

1. Introduction

2. THH for Spectral Categories and Shadows

3. Duality and trace

4. Morita equivalence in bicategories 14

5. Euler characteristics for base change objects 17

6. Example: Fixed Point Invariants 23

7. A $\pi_{0}$-level cyclotomic trace 26

Appendix A. Identifying $\mathscr{L} X^{f}$ via THH 30

References

\section{INTRODUCTION}

Many of the technical achievements of modern homotopy theory and algebraic geometry are motivated by questions arising from fixed point theory. Lefschetz's fixed point theorem is an incredibly successful application of cohomology theory, and it provides the intuition for Grothendieck's development of étale cohomology, via the Weil conjectures. Building on the Riemann-Roch theorem, the Atiyah-Singer index theorem [AS68] is in essence also a fixed point theorem. In each of these theorems, the goal is to obtain geometric information about fixed points from cohomological information. In this paper, we begin to relate the cyclotomic trace to fixed point theory, with topological Hochschild homology playing the role of the cohomology theory.

The most basic cohomological invariant of a self-map $f: X \rightarrow X$ is the Lefschetz number; it is a sort of twisted Euler characteristic. The Lefschetz number detects fixed points, but it is not a complete invariant. For that we need a more powerful invariant: the Reidemeister trace, defined as follows. Let $\left\{x_{1}, \ldots, x_{n}\right\}$ be the set of fixed points of $f$. We say $x_{i}$ and $x_{j}$ are in the same fixed point class if there is a path $\gamma$ from $x_{i}$ to $x_{j}$ such that $\gamma \simeq f(\gamma)$ relative $\left\{x_{i}, x_{j}\right\}=\left\{f\left(x_{i}\right), f\left(x_{j}\right)\right\}$. This is an equivalence relation which partitions the set of fixed points into fixed point classes, and the free abelian group on fixed point classes is denoted $\mathbf{Z}\left[\pi_{1}(X)_{f}\right]$. The Reidemeister trace of $f$ is

Date: 14th Aug, 2018.

2010 Mathematics Subject Classification. 16D90, 18D05, 19D55, 55M20, 55R12. 
$R(f)=\sum_{x_{i}}$ ind $\left(x_{i}\right)\left[x_{i}\right] \in \mathbf{Z}\left[\pi_{1} X_{f}\right]$. We then have $L(f)=\sum_{x_{i}}$ ind $\left(x_{i}\right)$. The Reidemeister trace is a more refined invariant than the Lefschetz number since it supports a converse to the Lefschetz fixed point theorem [Gh66, Wec41].

From the perspective of homotopy theory, this description of the Reidemeister trace is unsatisfying. There are many reasons for this. One is that in this formulation the Reidemeister trace appears to be a strange combination of unstable and stable data. This can be resolved by recognizing that the Reidemeister trace is a map of spectra

$$
S \rightarrow \Sigma_{+}^{\infty} \mathscr{L} X^{f},
$$

where $\mathscr{L} X^{f}$ is the space of paths $x \rightarrow f(x)$ [Pon10, Pon16].

Experience with algebraic $K$-theory makes the above formulation of the Reidemeister trace very suggestive. Algebraic $K$-theory is a universal receptacle for Euler characteristics [Wal85, Bar16, BGT13], and it comes equipped with the "cyclotomic trace" map $K(R) \rightarrow \mathrm{THH}(R)$, where the target is an invariant known as topological Hochschild homology [BHM93]. For a topological space $X$, the algebraic $K$-theory of $X$ is defined to be $K\left(\sum_{+}^{\infty} \Omega X\right)$, and $\pi_{0} K\left(\sum_{+}^{\infty} \Omega X\right)$ contains a canonical element $[X]$ corresponding to $X$. It is a folk theorem that the composition

$$
S \stackrel{[X]}{\longrightarrow} K\left(\Sigma_{+}^{\infty} \Omega X\right) \stackrel{\operatorname{tr}}{\rightarrow} \mathrm{THH}\left(\Sigma_{+}^{\infty} \Omega X\right) \simeq \Sigma_{+}^{\infty} \mathscr{L} X \rightarrow S
$$

is the Euler characteristic.

The appearance of the loop space and Euler characteristic strongly suggests that the "twisted Euler characteristic" $R(f)$ should arise in a very similar way, and there should be corresponding higher traces. In future work we show that indeed, $R(f)$ is in the image of some cyclotomic trace. The main step in showing that is completed in this paper.

Theorem 1.1. Let $X$ be a topological space homotopy equivalent to a finite $C W$-complex. The Reidemeister trace is naturally equivalent to the THH transfer

$$
\mathrm{THH}\left(\operatorname{Mod}_{S}^{c}\right) \rightarrow \mathrm{THH}\left(\operatorname{Mod}_{\Sigma_{+}^{\infty} \Omega X}^{c}\right) \rightarrow \mathrm{THH}\left(\operatorname{Mod}_{\Sigma_{+}^{\infty} \Omega X}^{c} ; F\right) .
$$

In this statement $\operatorname{Mod}_{A}^{c}$ is the category of compact $A$ modules. The object $\mathrm{THH}\left(\operatorname{Mod}_{A}^{c} ; F\right)$ is a twisted variant of THH (Definition 2.9).

The equivalence referenced in Theorem 1.1 is induced by Morita equivalences, which are maps

$$
\mathrm{THH}(A) \stackrel{\sim}{\rightarrow} \mathrm{THH}\left(\operatorname{Mod}_{A}^{c}\right) .
$$

In this direction the map is not hard to define, but the homotopy inverse is far less obvious. It would be desirable to know the inverse. We give a reasonably description of the inverse, and give a very explicit description on $\pi_{0}$.

For a ring spectrum $A$, an endomorphism $f: M \rightarrow M$ of a compact $A$-module spectrum determines a map $S \rightarrow \operatorname{End}(M)$. Composing with the inclusion of the zero skeleton defines a map

$$
S \rightarrow \operatorname{End}(M) \rightarrow \mathrm{THH}\left(\operatorname{Mod}_{A}^{c}\right)
$$

and so an element $[f] \in \pi_{0} \operatorname{THH}\left(\operatorname{Mod}_{A}^{c}\right)$. This sets up the second main theorem of this paper.

Theorem 1.2. The image of $[f] \in \pi_{0} \mathrm{THH}\left(\operatorname{Mod}_{A}^{c}\right)$ under the Morita invariance isomorphism

$$
\mathrm{THH}\left(\operatorname{Mod}_{A}^{c}\right) \simeq \mathrm{THH}(A)
$$

is the bicategorical trace of $f$. In particular, for a module $M \in \operatorname{Mod}_{A}^{c}$, the image of $\left[\operatorname{id}_{M}\right]$ is $\chi(M)$. 


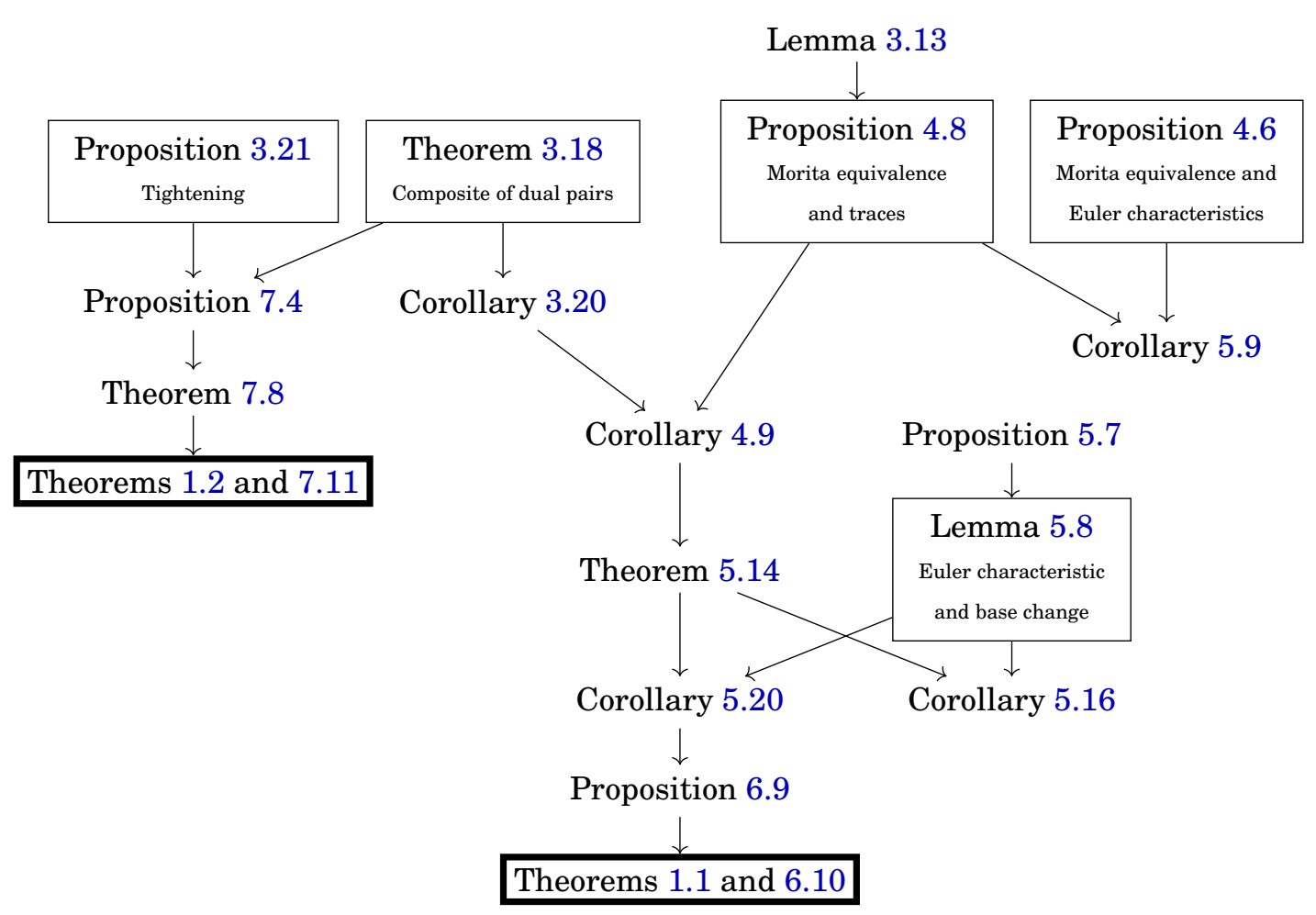

FIGURE 1.3. Concordance of results

The bicategorical trace is defined in Definition 3.8.

As indicated above, all of these invariants are generalizations of the Euler characteristic and, less obviously, they share many formal properties. This observation provides a conceptually clean and very general approach to both Theorems 1.1 and 1.2: duality, shadows and traces in bicategories [Pon10, PS13] exactly capture the relevant properties of the Euler characteristic and its generalizations. Then these theorems are special cases of far more general results that are proven without any reference to a particular bicategory.

The relevant bicategorical theoretic machinery is developed or recalled in the body of the paper. The key foundational concepts are

- base change objects (see Definition 2.7).

- the trace (see Definition 3.8)

- the Euler characteristic (see Definition 3.15)

- Morita equivalences (see Section 4)

Every theorem in this paper studies the interplay between some of these ingredients. For the convenience of the reader, we provide a concordance of these results in Figure 1.3, so that they may see the logical dependencies. The four boxed theorems at the top of the figure are the results from which all of the results in this paper follow. The logical progression is one of gradual specialization - the difficulty is in identifying the correct categorical context for proving the main results, not in the category theory itself.

1.1. Outline. In Section 2 we establish some results about the bicategory of spectral categories, and how THH behaves on these. We prove that THH is a shadow in the sense of [Pon10]. In Section 3 we define traces and Euler characteristics in bicategories. 
In the bicategorical context, the Euler characteristic is an invariant of 1-cells, and we establish a number of results about the composition of these characteristics.

Section 4 and Section 5 are devoted to the properties of traces and Euler characteristics under Morita invariance. These are the technical core of the paper, and every result needed to address the main example is treated in great generality in these sections. Section 4 addresses how traces behave with respect to Morita invariance, while Section 5 discusses how bicategorical Euler characteristics behave under certain base change maps.

Having related shadows and THH, in Section 6 we relate the transfer in THH to the Reidemeister trace. This is achieved by observing that transfers in THH are nothing more than an example of base change. The results from Section 4 and Section 5 then allow us to very explicitly identify certain transfers.

In Section 7 we show that the "inclusion of objects" map on THH is exactly computed by the bicategorical trace, finally relating the two notions of trace that arise in the literature.

A crucial, but lengthy, computation is relegated to the appendix.

1.2. Bicategories and Notation. Here we set our definitions and notations for bicategories. For much more thorough treatments see [Bén67, Lei]. A bicategory $\mathscr{B}$ consists of objects, $A, B, \ldots$, called 0 -cells, and categories $\mathscr{B}(A, B)$ for each pair of objects $A, B$. Objects in the category $\mathscr{B}(A, B)$ are called 1-cells and morphisms are called 2-cells. The unit 1-cell associated to a 0-cell $A$ is denoted $U_{A}$. There are horizontal composition functors

$$
\odot: \mathscr{B}(A, B) \times \mathscr{B}(B, C) \rightarrow \mathscr{B}(A, C) .
$$

They need not be strictly associative or unital.

The most illuminating examples of bicategories for this paper are:

- The bicategory whose 0-cells are rings and, for rings $A$ and $B, \mathscr{B}(A, B)$ is the category of $(A, B)$-bimodules. The horizontal composition is the tensor product.

- The bicategory whose 0-cells are spaces and, for spaces $A$ and $B, \mathscr{B}(A, B)$ is the category of spaces over $A \times B$. The horizontal composition is the pullback along the diagonal. This bicategory also has a stable version [MS06].

1.3. Acknowledgments. This paper should be regarded as a step in manifesting a perspective linking fixed point theory, $K$-theory, and topological Hochschild homology that has long been known to experts like Randy McCarthy, John Klein, and Bruce Williams. Parts of this perspective have appeared explicitly in the unpublished thesis of Iwashita [Iwa99].

Campbell thanks Randy McCarthy for a useful conversation about $K$-theory and fixed point theory. He also thanks Ralph Cohen for teaching him the ubiquity and utility of the free loop space. Ponto was partially supported by a Simons Collaborations Grant.

We are especially appreciative of the efforts of a very helpful referee who insights significantly improved this paper.

\section{THH FOR Spectral CATEgORIES AND SHADOWs}

In this section we define topological Hochschild homology and review the properties of spectral categories that are useful for our main applications. We show that THH, as an invariant of spectrally enriched categories, is a shadow in the sense of [Pon10]. This allows us to work in the generality of bicategories, easing and clarifying proofs and simplifying later work.

As we will make clear, the natural home for THH is the bicategory of spectral categories. The other familiar property of THH, Morita invariance, is a consequence of this 
structure. We emphasize that for proving general theorems about THH almost no other structure is used except for that provided by shadows. There is precedent for this viewpoint in the literature. In [BM12] the authors essentially manipulate THH as a shadow. As another example, in order to explore formal properties of Hochschild homology of DG-categories, Kaledin in [Kal15] defines "trace functors" and then notes that they are similar to the second-named author's shadows. From this perspective, there is in some sense nothing "special" about THH. Of course, its main property is that it receives a map from algebraic $K$-theory, but we are not yet using that structure.

Topological Hochschild homology is defined at varying levels of generality: it can be defined for ring spectra [Bök], rings with a bimodule coordinate [DM96], spectral categories and spectral categories with a bimodule coordinate [BM12]. For the moment, we work in the generality of spectral categories and bimodules. We begin by considering spectral categories enriched in either symmetric or orthogonal spectra [MMSS01].

Definition 2.1. A spectral category $\mathscr{C}$ is pointwise cofibrant if each mapping spectrum $\mathscr{C}(a, b)$ is cofibrant in the enriching category.

Definition 2.2. Let $\mathscr{C}$ be a spectral category. Then a $\mathscr{C}$-module is a spectral functor $\mathscr{C} \rightarrow$ Sp.

Definition 2.3. A $(\mathscr{C}, \mathscr{D})$-bimodule is a functor $\mathscr{M}: \mathscr{C}^{\mathrm{op}} \wedge \mathscr{D} \rightarrow \mathrm{Sp}$. That is, $\mathscr{M}$ is a collection of spectra $\mathscr{M}(c, d)$ for $c \in \mathscr{C}, d \in \mathscr{D}$ together with maps

$$
\begin{aligned}
& \mathscr{C}\left(c, c^{\prime}\right) \wedge \mathscr{M}\left(c^{\prime}, d\right) \rightarrow \mathscr{M}(c, d) \\
& \mathscr{M}(c, d) \wedge \mathscr{D}\left(d, d^{\prime}\right) \rightarrow \mathscr{M}\left(c, d^{\prime}\right)
\end{aligned}
$$

An $(\mathscr{C}, \mathscr{D})$-bimodule is pointwise cofibrant if $\mathscr{M}(c, d)$ is cofibrant. A morphism of $(\mathscr{C}, \mathscr{D})$-bimodules $\mathscr{M} \rightarrow \mathscr{M}^{\prime}$ is a collection of maps $\mathscr{M}(c, d) \rightarrow \mathscr{M}^{\prime}(c, d)$ which commute with the appropriate structure.

Remark 2.4. Note that this has the opposite variance of what is standard for bimodules in the literature. This convention seems to be more useful for bookkeeping for us.

Definition 2.5. Let $\mathscr{C}$ be a pointwise cofibrant spectral category, and $\mathscr{Q}$ a $(\mathscr{C}, \mathscr{C})$-bimodule. The topological Hochschild homology of $\mathscr{C}$ with coefficients in $\mathscr{Q}$ is the geometric realization of a spectrum whose $n$th simplicial level is

$$
\operatorname{THH}(\mathscr{C} ; \mathscr{Q})_{n}:=N_{n}^{\mathrm{cy}}(\mathscr{C}, \mathscr{Q})=\bigvee_{c_{0}, \ldots, c_{n}} \mathscr{C}\left(c_{0}, c_{1}\right) \wedge \mathscr{C}\left(c_{1}, c_{2}\right) \wedge \cdots \wedge \mathscr{C}\left(c_{n-1}, c_{n}\right) \wedge \mathscr{Q}\left(c_{n}, c_{0}\right)
$$

That is,

$$
\operatorname{THH}(\mathscr{C} ; \mathscr{Q}):=\mid \operatorname{THH}(\mathscr{C} ; \mathscr{Q}) \text {.| }
$$

Remark 2.6. The reader is warned that most literature makes a distinction between Bökstedt's construction $\mathrm{THH}(\mathscr{C} ; \mathscr{Q})$ and $N^{\mathrm{cy}}(\mathscr{C} ; \mathscr{Q})$. When $\mathscr{C}$ is pointwise cofibrant, they are equivalent. Since we will work on the level of homotopy categories, and ignore questions of equivariance, we therefore ignore the distinction.

Spectral bimodules may be manufactured from functors. This is an example of what we later call base change.

Definition 2.7. Let $F: \mathscr{A} \rightarrow \mathscr{C}$ and $G: \mathscr{B} \rightarrow \mathscr{C}$ be functors between spectral categories. Define an $(\mathscr{A}, \mathscr{B})$-bimodule ${ }_{F} \mathscr{C}_{G}$ as follows. For objects $a \in \mathscr{A}$ and $b \in \mathscr{B}$

$$
{ }_{F} \mathscr{C}_{G}(a, b):=\mathscr{C}(F(a), G(b)) .
$$

The right action of $\mathscr{B}$ on ${ }_{F} \mathscr{C}_{G}$ is given by the functor $G$ :

$$
\begin{aligned}
F \mathscr{C}_{G}(a, b) \wedge \mathscr{B}\left(b, b^{\prime}\right) & =\mathscr{C}(F(a), G(b)) \wedge \mathscr{B}\left(b, b^{\prime}\right) \rightarrow \mathscr{C}(F(a), G(b)) \wedge \mathscr{C}\left(G(b), G\left(b^{\prime}\right)\right) \\
& \rightarrow \mathscr{C}\left(F(a), G\left(b^{\prime}\right)\right)={ }_{F} \mathscr{C}_{G}\left(a, b^{\prime}\right)
\end{aligned}
$$


The left action of $\mathscr{A}$ is similar.

Example 2.8. When $F: \mathscr{C} \rightarrow \mathscr{C}$ is an endofunctor and $G=\mathrm{id}$, we can form ${ }_{F} \mathscr{C}$, and similarly $\mathscr{C}_{F}$.

Definition 2.9. Let $\mathscr{C}$ be a pointwise cofibrant spectral category and $F: \mathscr{C} \rightarrow \mathscr{C}$ be an endofunctor. We defined the right twisted topological Hochschild homology to be

$$
\operatorname{THH}(\mathscr{C} ; F):=\operatorname{THH}\left(\mathscr{C} ; \mathscr{C}_{F}\right)
$$

Remark 2.10. When $F=\mathrm{id}$, we recover $\mathrm{THH}(\mathscr{C})$.

Example 2.11. A good example to keep in mind is the following. Let $A$ be a commutative ring spectrum, $P$ be an $A$-module, and let $\operatorname{Mod}_{A}^{c}$ denote the category of compact $A$ modules. Consider the functor $-\wedge_{A} P: \operatorname{Mod}_{A}^{c} \rightarrow \operatorname{Mod}_{A}^{c}$ given by $M \mapsto M \wedge_{A} P$, where $M \in \operatorname{Mod}_{A}^{c}$. We show in Example 5.12 that the twisted $\operatorname{THH}\left(\operatorname{Mod}_{A}^{c} ;-\wedge_{A} P\right)$ coincides with $\operatorname{THH}(A ; P)$.

Topological Hochschild homology is clearly functorial in the bimodule coordinate so that given a map of $(\mathscr{C}, \mathscr{C})$-bimodules $\mathscr{Q} \rightarrow \mathscr{Q}^{\prime}$ there is a map

$$
\operatorname{THH}(\mathscr{C} ; \mathscr{Q}) \rightarrow \operatorname{THH}\left(\mathscr{C} ; \mathscr{Q}^{\prime}\right) \text {. }
$$

Furthermore, if $\mathscr{A} \rightarrow \mathscr{C}$ is a map, then we get an induced map $\operatorname{THH}(\mathscr{A}) \rightarrow \operatorname{THH}(\mathscr{C})$. There is also a refinement of both [BM12]. Let $F: \mathscr{A} \rightarrow \mathscr{C}$ be a map of spectral categories and let $\mathscr{Q}$ be a $(\mathscr{C}, \mathscr{C})$-bimodule. Then there is a map

$$
\operatorname{THH}\left(\mathscr{A} ;{ }_{F} \mathscr{Q}_{F}\right) \rightarrow \operatorname{THH}(\mathscr{C}, \mathscr{Q})
$$

and if there is a map $\mathscr{P} \rightarrow{ }_{F} \mathscr{Q}_{F}$ we obtain

$$
\mathrm{THH}(\mathscr{A} ; \mathscr{P}) \rightarrow \mathrm{THH}(\mathscr{C}, \mathscr{Q}) .
$$

We now describe the bicategory structure on the category of spectral categories. First, we note some homotopical properties of spectral categories. In the sequel, we work with a bicategory enriched in various homotopy categories; the following remarks establish that we may do this.

To begin, we have the following rephrasing of [SS03, Prop. 6.1] found in [BM12, Prop. 2.4].

Proposition 2.12. The category, $\operatorname{Mod}_{\mathscr{C}, \mathscr{D})}$ of $(\mathscr{C}, \mathscr{D})$-bimodules forms a closed model category with object-wise weak equivalences.

For any small spectral category $\mathscr{C}$, we have the following rephrasing of [SS03, Prop. 6.3] due to [BM12, Prop. 2.7, Prop. 2.8].

Proposition 2.13. Given a small spectral category $\mathscr{C}$ there is an endofunctor $Q: \mathscr{C}$ at $_{S p} \rightarrow$ $\mathscr{C}$ at $t_{S p}$ such that $Q \mathscr{C}$ is pointwise cofibrant and there is a map $Q \mathscr{C} \rightarrow \mathscr{C}$ that is a pointwise weak equivalence. Furthermore, if $\mathscr{M}$ is a cofibrant $(\mathscr{C}, \mathscr{D})$-module, then $\mathscr{M}$ is pointwise cofibrant.

Furthermore, by the remark following [BM12, Prop. 3.6], if $\mathscr{C}$ is pointwise cofibrant, and $\mathscr{P} \rightarrow \mathscr{P}^{\prime}$ is a weak equivalence of spectral categories, then the induced map

$$
\mathrm{THH}(\mathscr{C}, \mathscr{P}) \rightarrow \operatorname{THH}\left(\mathscr{C}, \mathscr{P}^{\prime}\right)
$$

is a weak equivalence. Thus, for instance, if $Q \mathscr{P} \rightarrow \mathscr{P}$ is a cofibrant replacement of $\mathscr{P}$, $\mathrm{THH}(\mathscr{C}, Q \mathscr{P}) \rightarrow \mathrm{THH}(\mathscr{C}, \mathscr{P})$ is a weak equivalence.

These propositions imply that can move between models and replace bimodules by weakly equivalent ones at will. Given this, we work on the level of homotopy categories. 
Definition 2.14. The bicategory of small spectral categories is the bicategory whose objects are pointwise cofibrant small spectral categories, and whose morphism categories are

$$
\operatorname{Ho}\left(\operatorname{Mod}_{(\mathscr{C}, \mathscr{D})}\right)
$$

for pointwise cofibrant small spectral categories $\mathscr{C}$ and $\mathscr{D}$.

Remark 2.15. The composition of 1-cells is defined as follows. Let $\mathscr{M}$ be an $(\mathscr{C}, \mathscr{D})$ bimodule and $\mathscr{N}$ an $(\mathscr{D}, \mathscr{E})$-bimodule. Then we may form an $(\mathscr{C}, \mathscr{E})$-bimodule $\mathscr{M} \odot \mathscr{N}$

$$
\begin{aligned}
(\mathscr{M} \odot \mathscr{N})(c, e) & :=\mathscr{M}(c,-) \wedge \stackrel{\mathbf{L}}{\mathscr{N}}(-, e) \\
& :=B(\mathscr{M}(c,-), \mathscr{D}, \mathscr{N}(-, e))
\end{aligned}
$$

where $B(-,-,-)$ denotes the two-sided bar construction.

This descends to

$$
\operatorname{Ho}\left(\operatorname{Mod}_{(\mathscr{C}, \mathscr{D})}\right) \times \operatorname{Ho}\left(\operatorname{Mod}_{(\mathscr{D}, \mathscr{E})}\right) \rightarrow \operatorname{Ho}\left(\operatorname{Mod}_{(\mathscr{C}, \mathscr{E})}\right)
$$

Checking that this is associative is straightforward but tedious. One explicitly writes out the bar construction and cofibrantly replaces as needed. The composition of 2-cells is the composition of natural transformations.

As a cyclic bar construction, THH has cyclic invariance built into it. This cyclic invariance is also present in Hochschild homology and is an essential part of the HattoriStallings trace

$$
K_{0}(A) \rightarrow H H_{0}(A) .
$$

There is a general categorical setup due to the second named author [Pon10] that encodes exactly the kind of properties that THH enjoys as a functor of spectral categories.

Definition 2.16 ([Pon10]). Let $\mathscr{B}$ be a bicategory. A shadow functor for $\mathscr{B}$ consists of functors

$$
\langle-\rangle: \mathscr{B}(C, C) \rightarrow \mathbf{T}
$$

for each object $C$ of $\mathscr{B}$ and some fixed category $\mathbf{T}$, equipped with a natural isomorphism

$$
\theta:\langle M \odot N\rangle \stackrel{\cong}{\rightarrow}\langle N \odot M\rangle
$$

for $M \in \mathscr{B}(C, D)$ and $N \in \mathscr{B}(D, C)$ such that the following diagrams commute whenever they make sense:

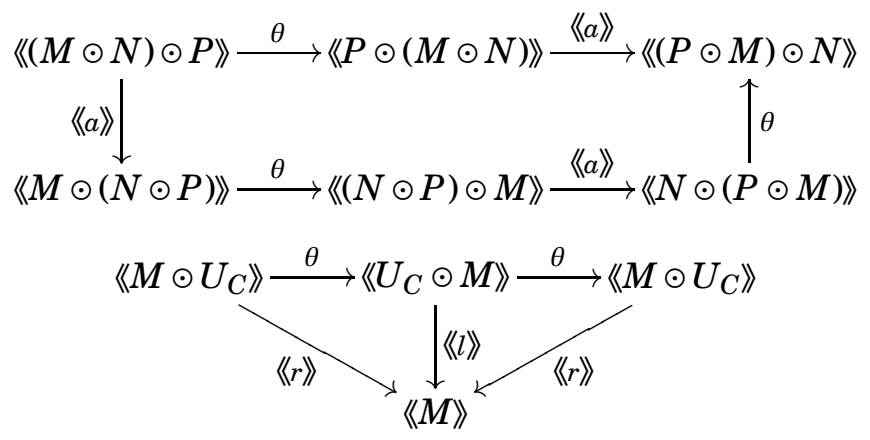

Note that if $\langle-\rangle$ is a shadow functor on $\mathscr{B}$, then the composite

$$
\langle M \odot N\rangle \stackrel{\theta}{\longrightarrow}\langle\langle N \odot M\rangle \stackrel{\theta}{\longrightarrow}\langle M M \odot N\rangle
$$

is the identity [PS13, Prop. 4.3].

Theorem 2.17. Topological Hochschild Homology is a shadow. That is, it gives a family of functors

$$
\mathrm{THH}(-): \operatorname{Ho}\left(\operatorname{Mod}_{(\mathscr{C}, \mathscr{C})}\right) \rightarrow \mathrm{Ho}(\mathrm{Sp})
$$

that satisfy the required properties. 
Proof. The main property of shadows is that for $(\mathscr{C}, \mathscr{D})$-bimodule $\mathscr{M}$ and a $(\mathscr{D}, \mathscr{C})$-bimodule $\mathscr{N}$, there is an isomorphism

$$
\theta: 《 \mathscr{M} \odot \mathscr{N} 》 \rightarrow\langle\mathscr{N} \odot \mathscr{M} 》 .
$$

Unpacking this into the usual notation, this is equivalent to the demand that there is an isomorphism

$$
\theta: \operatorname{THH}(\mathscr{C}, B(\mathscr{M}, \mathscr{D}, \mathscr{N})) \rightarrow \operatorname{THH}(\mathscr{D}, B(\mathscr{N}, \mathscr{C}, \mathscr{M}))
$$

However, this is the classical Dennis-Morita-Waldhausen argument [BM12, Prop. 6.2]

- in this case, there is an isomorphism of underlying point-set spectra.

The commutativity of the rest of the diagrams follow from essentially the same argument.

\section{Duality AND TRACE}

In the previous section, we showed that THH is an example of a shadow on a bicategory. This is quite a general notion, and many bicategories possess shadows. In addition, in any bicategory with shadow, one can define a notion of trace, which one can think of as a vast generalization of the trace of an endomorphism in a symmetric monoidal category. In this section we recall the definitions required to define a trace and collect the results about the trace that we will need below. It is at this point that we begin to work in bicategorical generality.

As a starting point it is useful to have a few bicategories in mind. The following two examples are very important for our intended applications. Let $\mathscr{V}$ be a symmetric monoidal category. (In what follows, one can imagine that $\mathscr{V}$ is the category of spectra.)

$i$. Let $\mathscr{B}(\operatorname{Mon}(\mathscr{V}))$ be the bicategory whose objects are monoids in $\mathscr{V}, 1$-cells are bimodules over monoids in $\mathscr{V}$ and whose 2-cells are maps of bimodules.

ii. Let $\mathscr{B}(\mathbf{C a t}(\mathscr{V}))$ be the bicategory whose objects are categories enriched in $\mathscr{V}$,

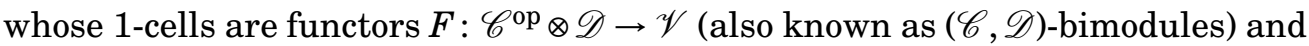
whose 2-cells are natural transformations of such.

These bicategories both have shadows that take values in $\mathscr{V}$.

Example 3.1. Let $\mathscr{V}=\mathbf{S p}$. Then $\mathscr{B}(\mathbf{C a t}(\mathscr{V}))$ is the bicategory of spectral categories. Because of the homotopical issues outlined above we work in the full subcategory of pointwise cofibrant small spectral categories. In general, if $\mathscr{V}$ and $\operatorname{Cat}(\mathscr{V})$ have some kind of homotopical structure, we understand $\mathscr{B}(\mathbf{C a t}(\mathscr{V}))$ to be modified in order to give the homotopically correct definitions.

Remark 3.2. Note that every monoid in $\mathscr{V}$ can be made into a $\mathscr{V}$-category with one object, giving an embedding $\operatorname{Mon}(\mathscr{V}) \rightarrow \operatorname{Cat}(\mathscr{V})$; this is the enriched version of the usual embedding Mon $\rightarrow$ Cat. Thus, at the level of bicategories, we have an embedding

$$
\mathscr{B}(\operatorname{Mon}(\mathscr{V})) \rightarrow \mathscr{B}(\operatorname{Cat}(\mathscr{V})) .
$$

The following definition is at the core of all of the constructions in this paper.

Definition 3.3. We say that a 1-cell $M \in \mathscr{B}(C, D)$ in a bicategory is right dualizable if there is a 1-cell $N \in \mathscr{B}(D, C)$, called its right dual, and coevaluation and evaluation 2-cells $\eta: U_{C} \rightarrow M \odot N$ and $\epsilon: N \odot M \rightarrow U_{D}$ satisfying the triangle identities. We say that $(M, N)$ is a dual pair, that $N$ is left dualizable, and that $M$ is its left dual.

Remark 3.5. While we will not use them as a formal proof, some of the results in the next sections have illuminating graphical descriptions as pasting diagrams.

In our pasting diagrams vertices represent 0 -cells, edges represent 1-cells, and colored regions represent 2-cells. Since we will need to eventually make circular diagrams 


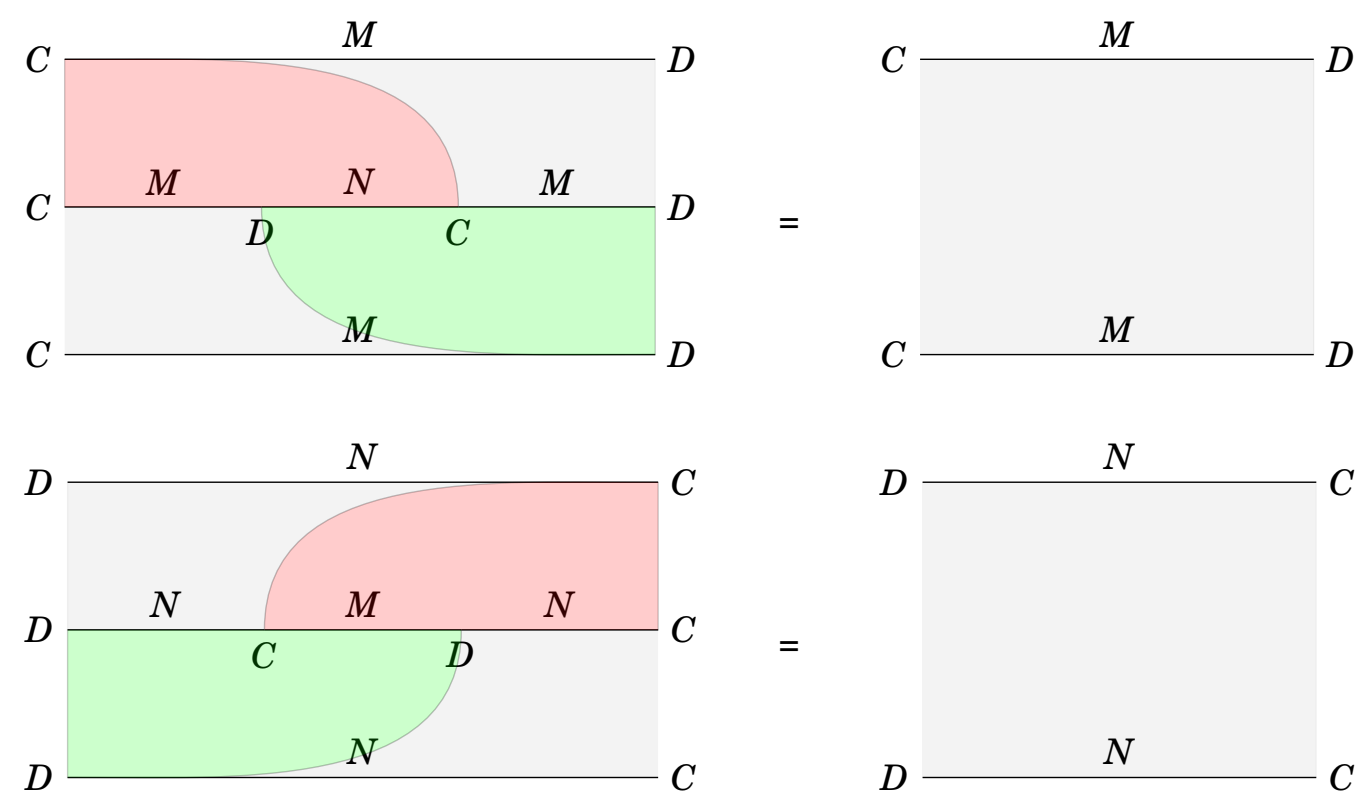

FiguRE 3.4. Pasting diagrams for dual pairs.

(Figure 3.10), we do not identify 0-cells when they are the same. Instead we rely on the convention that vertices in consecutive layers that align should be regarded as the same. We suppress unit isomorphisms and many unit 1-cells. Pale gray regions are identity 2-cells.

See Figure 3.4 for pasting diagrams for a dual pair.

Example 3.6. For rings $C$ and $D$, an $(C, D)$-bimodule $M$ is right dualizable if it is finitely generated and projective as a right $D$-module. A choice of dual is the $(D, C)$-bimodule of right $D$-module homomorphisms $\operatorname{Hom}_{D}(M, D)$. Note that $\operatorname{Hom}_{D}(M, D)$ is regarded as a $(D, C)$-bimodule using the left $C$-module structure on $M$ and left $D$-module structure on $D$.

The coevaluation map is the composite

$$
C \rightarrow \operatorname{Hom}_{D}(M, M) \stackrel{\sim}{\longleftarrow} M \otimes_{D} \operatorname{Hom}_{D}(M, D)
$$

where the second map is an isomorphism since $M$ is finitely generated and projective as an $D$ module. The evaluation map for this dual pair is the evaluation map

$$
\operatorname{Hom}_{D}(M, D) \otimes_{C} M \rightarrow D .
$$

Dually, $M$ is left dualizable if it is finitely generated and projective as a left $C$-module.

Example 3.7. Costenoble-Waner duality [MS06, Chapter 18] is a special case of the duality theory above and generalizes Spanier-Whitehead and Atiyah duality.

The parameterized stable homotopy category Ex of [MS06] has a fiberwise suspension spectrum functor from the bicategory of fibered spaces (without sections). If we regard a closed smooth manifold $X$, or compact ENR, as a space over $* \times X$ its fiberwise suspension spectrum is dualizable with dual the desuspension of the fiberwise one point compactification of the normal bundle [MS06, 18.5.1].

Using this definition and that of a shadow we can define traces of 2-cells associated to dualizable 1-cells. The following definition will be crucial for the constructions below. 


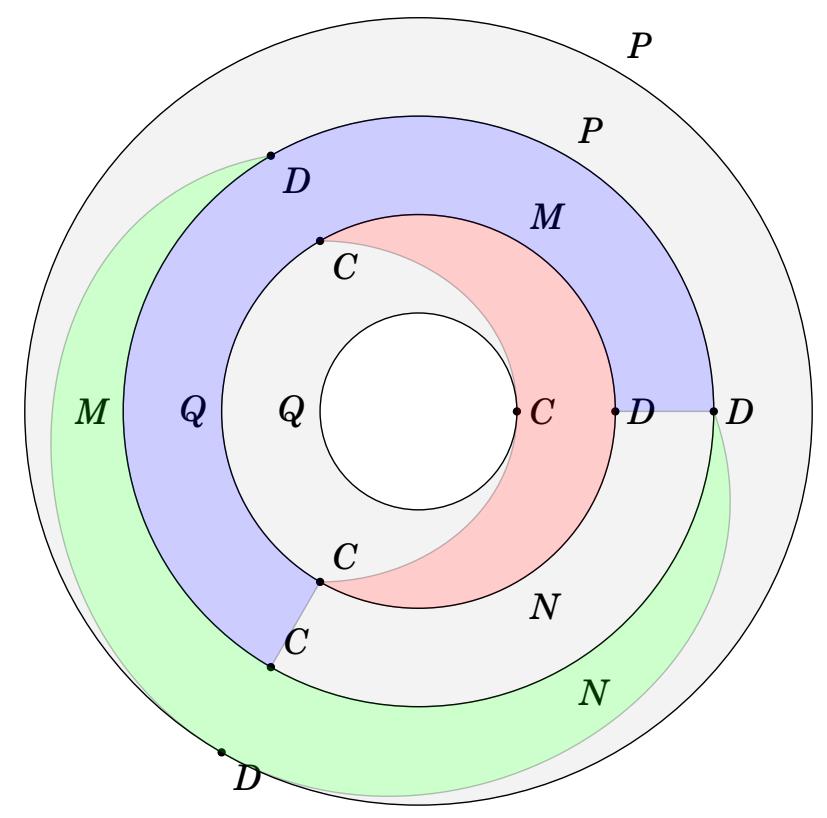

FiguRE 3.10. The trace

Definition 3.8. [Pon10] Let $\mathscr{B}$ be a bicategory with a shadow functor and $(M, N)$ be a dual pair. The trace of a 2 -cell $f: Q \odot M \rightarrow M \odot P$ is the composite:

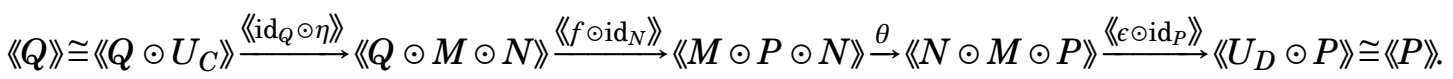

The trace of a 2-cell $g: N \odot Q \rightarrow P \odot N$ is

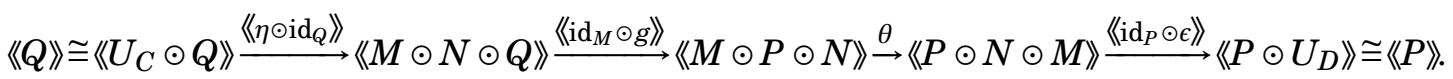

Remark 3.9. After applying the shadow, we glue together vertical edges to form a bullseye diagram as in Figure 3.10. As above, we do collapse most 0-cells. In these diagrams we read 2-cells as directed from the innermost circle to the outermost circle and 1-cells clockwise.

Once we have applied the shadow we compose 2-cells by stacking circles.

The bicategorical trace generalizes both the symmetric monoidal trace [DP80] and the Hattori-Stallings trace [Hat65, Sta65].

Remark 3.11. In [DP80], there is a particularly elegant proof of the Lefschetz fixed point theorem that relies on the observations that the fixed point index [Dol65] is the trace in the stable homotopy category, the Lefschetz number is the trace in the homotopy category of chain complexes, and the symmetric monoidal trace is functorial: that is

$$
F(\operatorname{tr}(f))=\operatorname{tr}(F(f)) .
$$

The Reidemeister trace, in its many variants, is an example of the bicategorical trace [Pon10]. Since these are bicategorical traces, the identification of the varied forms of the Reidemeister trace is a consequence of the functoriality of the bicategorical trace. 
Let $(M, N)$ be a dual pair and $Q$ and $P$ be 1-cells so that $N \odot Q \odot M$ and $M \odot P \odot N$ are defined. We then fix the following notation:

$$
\begin{gathered}
\eta_{Q}^{(M, N)}: Q \odot M \cong U_{C} \odot Q \odot M \stackrel{\eta \odot \operatorname{id}_{Q} \odot \operatorname{id}_{M}}{\longrightarrow} M \odot N \odot Q \odot M \\
\epsilon_{P}^{(M, N)}: M \odot P \odot N \odot M \stackrel{\operatorname{id}_{M} \odot \operatorname{id}_{P} \odot \epsilon}{\longrightarrow} M \odot P \odot U_{D} \cong M \odot P \\
{ }^{(M, N)} \eta_{Q}: N \odot Q \cong N \odot Q \odot U_{C} \stackrel{\operatorname{id}_{N} \odot \operatorname{id}_{Q} \odot \eta}{\longrightarrow} N \odot Q \odot M \odot N \\
{ }^{(M, N)} \epsilon_{P}: N \odot M \odot P \odot N \stackrel{\epsilon \odot \operatorname{id}_{P} \odot \operatorname{id}_{N}}{\longrightarrow} U_{D} \odot P \odot N \cong P \odot N
\end{gathered}
$$

Remark 3.12. If $(M, N)$ is a dual pair, the dual of a map $g: N \odot Q \rightarrow P \odot N$, denoted $g^{\star}$, is the composite

$$
Q \odot M \stackrel{\eta_{Q}^{(M, N)}}{\longrightarrow} M \odot N \odot Q \odot M \stackrel{\operatorname{id}_{M} \odot g \odot \mathrm{id}_{M}}{\longrightarrow} M \odot P \odot N \odot M \stackrel{\epsilon_{P}^{(M, N)}}{\longrightarrow} M \odot P
$$

Since $\operatorname{tr}(g)=\operatorname{tr}\left(g^{\star}\right)$ [PS13, Prop. 7.6] we freely move between traces defined with respect to $M$ and those defined with respect to $N$.

Note that $\eta_{Q}^{(M, N)}$ is the dual of ${ }^{(M, N)} \eta_{Q}, \epsilon_{Q}^{(M, N)}$ is the dual of ${ }^{(M, N)} \epsilon_{Q}$, and

$$
\langle Q\rangle\rangle \stackrel{\operatorname{tr}\left(\eta_{Q}^{(M, N)}\right)=\operatorname{tr}\left({ }^{(M, N)} \eta_{Q}\right)}{\longrightarrow}\langle N \odot Q \odot M\rangle \text { and }\langle M \odot P \odot N\rangle \stackrel{\operatorname{tr}\left(\varepsilon_{P}^{(M, N)}\right)=\operatorname{tr}\left({ }^{(M, N)} \epsilon_{P}\right)}{\longrightarrow}\langle P\rangle
$$

Lemma 3.13. For a dual pair $(M, N)$ and 1-cells $Q$ and $P$ so that $N \odot Q \odot M$ and $M \odot P \odot N$ are defined, $\operatorname{tr}\left(\epsilon_{P}^{(M, N)}\right)$ is

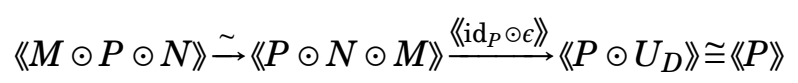

and $\operatorname{tr}\left(\eta_{Q}^{(M, N)}\right)$ is the composite

$$
\langle Q\rangle \cong\left\langle\left\langle Q \odot U_{C}\right\rangle \stackrel{\left\langle\operatorname{id}_{Q} \odot \eta\right\rangle}{\longrightarrow}\langle Q \odot \odot \odot \odot N\rangle \cong\langle\langle N \odot Q \odot M\rangle .\right.
$$

Proof. In the trace of $\epsilon_{P}^{(M, N)}$ (Figure 3.14a) there is a coevaluation/evaluation pair that can be canceled. Cancelling this pair gives the composite above and is illustrated in Figure 3.14b. The proof for $\eta_{Q}^{(M, N)}$ is similar.

The stable homotopy category is symmetric monoidal and the suspension spectrum of a closed smooth manifold or compact ENR $X$ is dualizable. The trace of the identity map of $X$ is a stable map $S \rightarrow S$ and this is the Euler characteristic of $X$ under the identification of stable $\pi_{0}$ with $\mathbb{Z}$. As a result, we refer to symmetric monoidal traces of identity maps and bicategorical traces of identity 2-cells as Euler characteristics and denote them by $\chi(X)$. We formalize this in a definition.

Definition 3.15. If $M \in \mathscr{B}(C, D)$ and $M$ is right dualizable, the Euler characteristic (Figure 3.17) is the trace of the identity 2-cell of $M$ and is a map

$$
\left\langle U_{C}\right\rangle \rightarrow\left\langle U_{D}\right\rangle \text {. }
$$

If $N$ is the right dual of $M, \chi(M)=\chi(N)$.

Remark 3.16. Thinking of the Euler characteristic as a map rather than a object is an important psychological move for working with constructions in the sequel. The importance of this formulation of the Euler characteristic cannot be overstated.

The Euler characteristic is multiplicative on fibrations and its refinements to the Lefschetz number and Reidemeister trace satisfy the appropriate generalizations of multiplicativity [PS14]. These results are consequences of the following very convenient result describing the compatibility between traces and bicategorical composition. It is 


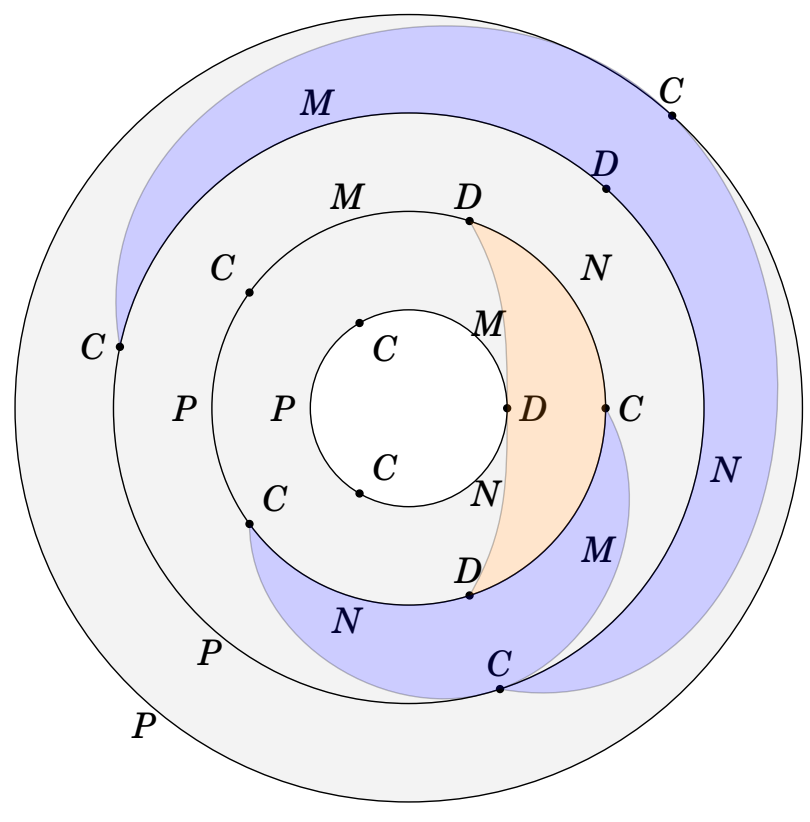

(A) The trace of $\epsilon_{P}^{(N, M)}$

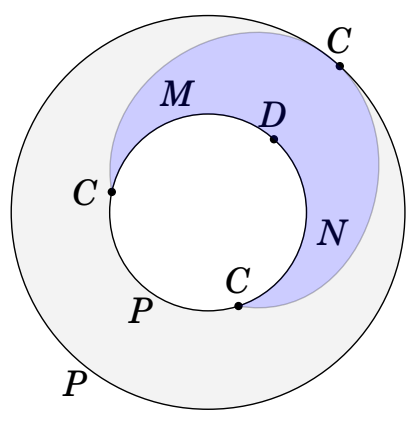

(B) After collapsing the coevaluation/evaluation pair

FiguRE 3.14. Diagrams for Lemma 3.13

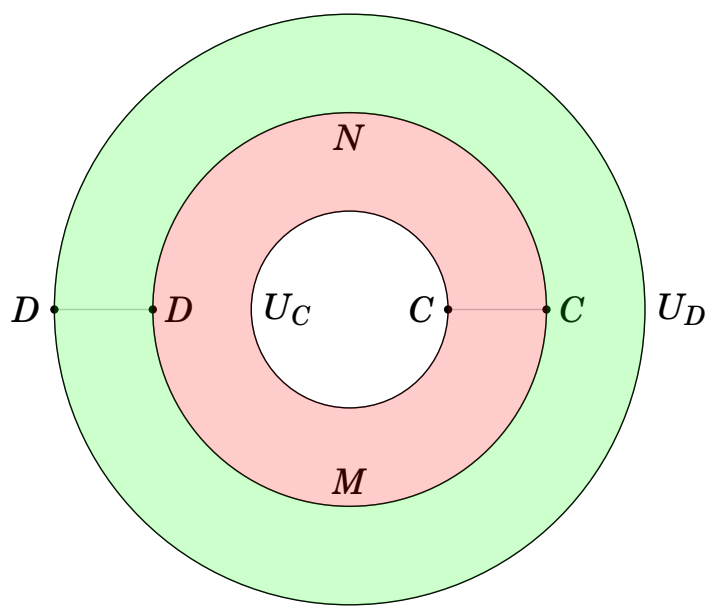

FIgURE 3.17. The Euler characteristic

an easy generalization of the corresponding symmetric monoidal fact and is an essential foundation for many of the results in the next sections.

Theorem 3.18. [MS06, 16.5.1][PS13, Prop. 7.5] If $M_{1} \in \mathscr{B}(C, D)$ and $M_{2} \in \mathscr{B}(D, E)$ are right dualizable, then $M_{1} \odot M_{2}$ is right dualizable. The trace of

$$
Q_{1} \odot M_{1} \odot M_{2} \stackrel{f_{1} \odot \operatorname{id}_{M_{2}}}{\longrightarrow} M_{1} \odot Q_{2} \odot M_{2} \stackrel{\operatorname{id}_{M_{1}} \odot f_{2}}{\longrightarrow} M_{1} \odot M_{2} \odot Q_{3}
$$




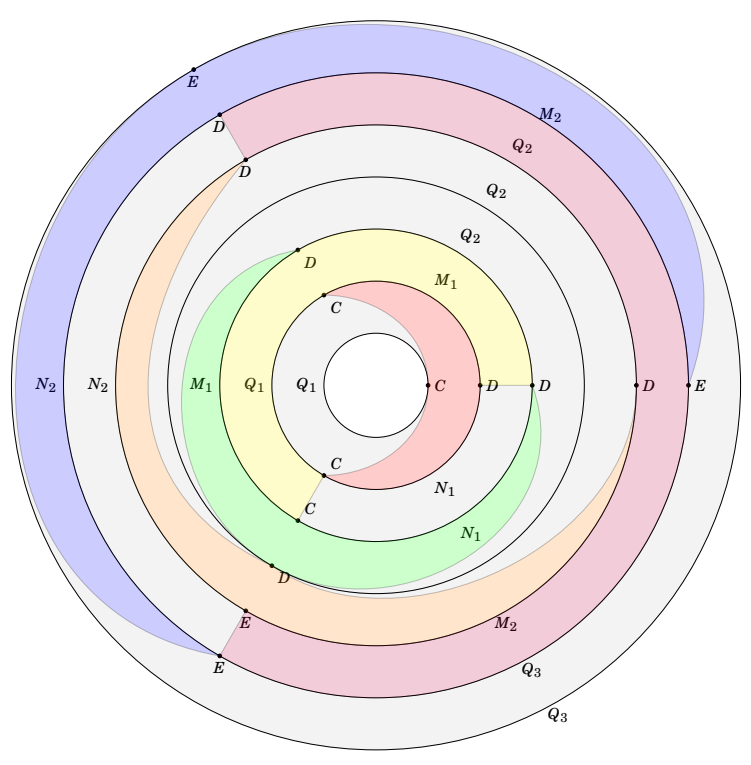

FiguRE 3.19. Composite of traces

$$
\left.\left\langle Q_{1}\right\rangle \stackrel{\operatorname{tr}\left(f_{1}\right)}{\longrightarrow}\left\langle Q_{2}\right\rangle \stackrel{\operatorname{tr}\left(f_{2}\right)}{\longrightarrow}\left\langle Q_{3}\right\rangle\right\rangle .
$$

Figure 3.19 is a graphical representation of the composite of traces. The trace of $\left(\operatorname{id}_{M_{1}} \odot f_{2}\right)\left(f_{1} \odot \operatorname{id}_{M_{2}}\right)$ can be visualized by sliding the outer colored segments over the inner light gray segments.

We will use Theorem 3.18 in the following form. Given dual pairs $\left(M_{1}, N_{1}\right)$ and $\left(M_{2}, N_{2}\right)$, a 1-cell $L$ so that $M_{1} \odot L \odot M_{2}$ is defined, and an endomorphism $Q \odot L \stackrel{f}{\rightarrow} L \odot P$, let $M_{1} \odot f \odot M_{2}$ be the composite

$$
\begin{aligned}
M_{1} \odot Q \odot N_{1} \odot M_{1} \odot L \odot M_{2} & \stackrel{\epsilon_{Q}^{\left(M_{1}, N_{1}\right)} \odot \mathrm{id}_{L} \odot \mathrm{id}_{M_{2}}}{\longrightarrow} M_{1} \odot Q \odot L \odot M_{2} \\
& \stackrel{\operatorname{id}_{M_{1}} \odot f \odot \mathrm{id}_{M_{2}}}{\longrightarrow} M_{1} \odot L \odot P \odot M_{2} \\
& \stackrel{\operatorname{id}_{M_{1}} \odot \mathrm{id}_{L} \odot \eta_{P}^{\left(M_{2}, N_{2}\right)}}{\longrightarrow} M_{1} \odot L \odot M_{2} \odot N_{2} \odot P \odot M_{2} .
\end{aligned}
$$

Corollary 3.20. If $L$ is right dualizable

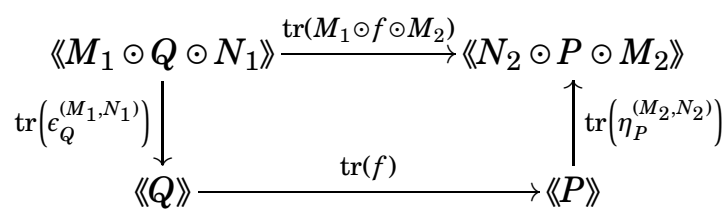

commutes. 
As the definition of $M_{1} \odot f \odot M_{2}$ suggests, this follows by applying Theorem 3.18 to the maps

$$
\begin{gathered}
M_{1} \odot Q \odot N_{1} \odot M_{1} \stackrel{\epsilon_{Q}^{\left(M_{1}, N_{1}\right)}}{\longrightarrow} M_{1} \odot Q \\
Q \odot L \stackrel{f}{\rightarrow} L \odot P \\
P \odot M_{2} \stackrel{\eta_{P}^{\left(M_{2}, N_{2}\right)}}{\longrightarrow} M_{2} \odot N_{2} \odot P \odot M_{2}
\end{gathered}
$$

Finally, we record a useful proposition that will be a needed on a few occasions. It is an easy consequence of the formal properties of the trace.

Proposition 3.21. [PS13, Prop. 7.1] Let $M$ be right dualizable, let $f: Q \odot M \rightarrow M \odot P$, $g: Q^{\prime} \rightarrow Q$ and $h: P \rightarrow P^{\prime}$ be 2-cells. Then

$$
\langle h\rangle \circ \operatorname{tr}(f) \circ\langle g\rangle\rangle=\operatorname{tr}\left(\operatorname{id}_{M} \odot h\right) \circ f \circ\left(g \odot \operatorname{id}_{M}\right) .
$$

\section{Morita Equivalence in Bicategories}

The Morita invariance of THH is one of its defining properties, and one of its most useful. If one takes the view that THH is a shadow on a bicategory, then the Morita invariance becomes a property not of THH itself, but rather its categorical context. That is, Morita equivalence is the natural notion of an equivalence in a bicategory, so THH is a Morita invariant simply because it is a bicategorical construct. Since everything we prove about Morita invariance is true at the level of bicategories, we work at that level of generality. This section recalls the definition of a Morita equivalence in a bicategory, and develops the basic properties of such equivalences with respect to trace and Euler characteristic. Since it is a notion of equivalence, the trace and characteristic are essentially insensitive to Morita equivalence, but keeping track of isomorphisms is important for the sequel and future work.

Definition 4.1. A pair of one cells $M \in \mathscr{B}(C, D)$ and $N \in \mathscr{B}(D, C)$ is a Morita equivalence if $(M, N)$ and $(N, M)$ are dual pairs and the coevaluation and evaluation maps for each dual pair are inverses. That is, if $\eta_{(M, N)}$ and $\epsilon_{(M, N)}$ are the coevaluation and evaluation for $(M, N)$ and $\eta_{(N, M)}$ and $\epsilon_{(N, M)}$ are the coevaluation and evaluation for $(N, M)$ then

$$
\begin{array}{ll}
\eta_{(N, M)} \circ \epsilon_{(M, N)}=\operatorname{id}_{N \odot M} & \epsilon_{(N, M)} \circ \eta_{(M, N)}=\operatorname{id}_{U_{C}} \\
\eta_{(M, N)} \circ \epsilon_{(N, M)}=\operatorname{id}_{M \odot N} & \epsilon_{(M, N)} \circ \eta_{(N, M)}=\operatorname{id}_{U_{D}}
\end{array}
$$

Definition 4.2. If $M \in \mathscr{B}(C, D)$ and $N \in \mathscr{B}(D, C)$ define a Morita equivalence, then $C$ and $D$ are said to be Morita equivalent.

Remark 4.3. Note that we use the dual pair as a subscript on the coevaluation and evaluation when there are multiple dual pairs. We will use a similar notation for the Euler characteristic.

Example 4.5. In the bicategory of rings, bimodules, and homomorphisms, a Morita equivalence is a $(C, D)$-bimodule $M$ and a $(D, C)$-bimodule $N$ so that $(M, N)$ and $(N, M)$ are dual pairs and, using the coevaluation and evaluation from these dual pairs,

$$
M \otimes_{D} N \cong C \quad \text { and } \quad N \otimes_{C} M \cong D .
$$

This is the familiar notion of Morita equivalence for rings and implies the functor

$$
N \otimes_{C}-: \operatorname{Mod}_{C} \rightarrow \operatorname{Mod}_{D}
$$

is equivalence of the category of (left) modules over $C$ and the category of (left) modules over $D$. 

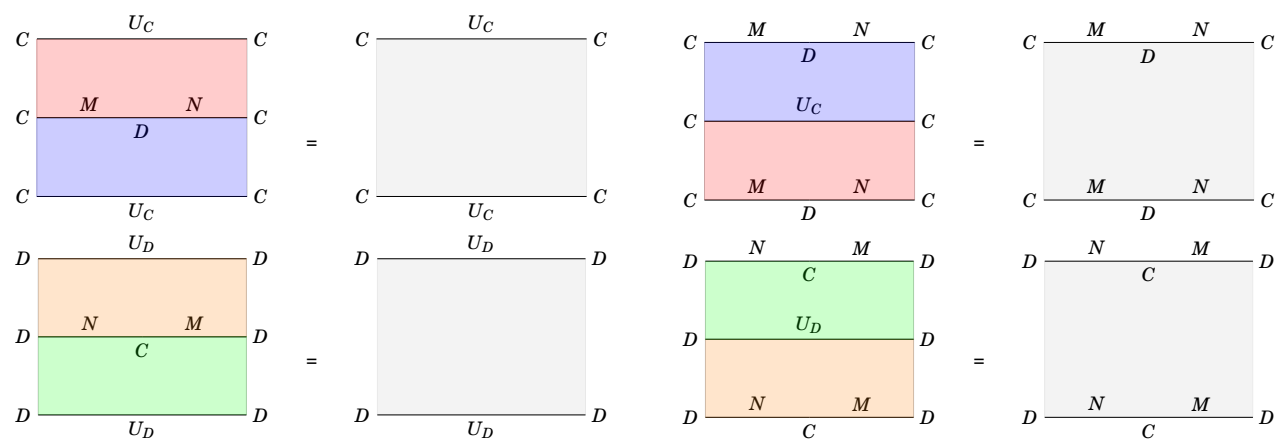

FIgURE 4.4. The diagrams for Morita equivalence. For one dual pair the coevaluation is red and the evaluation is green. For the other the coevaluation is orange and the evaluation is blue.

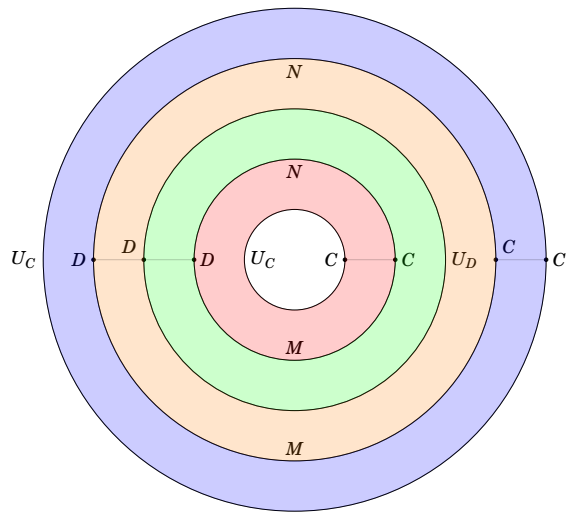

(A) The composite of Euler characteristics

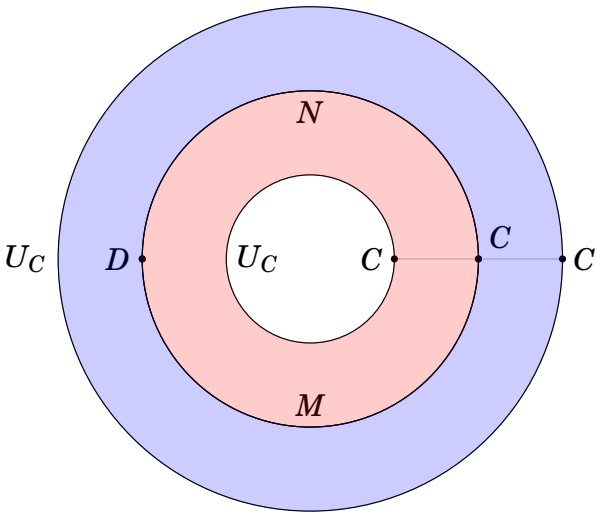

(B) After canceling the middle maps

FIGURE 4.7. Euler characteristics and Morita equivalence (Proposition 4.6)

The most familiar example of Morita equivalence is that the ring of $n$-by- $n$ matrices with elements in $C$ is Morita equivalent to $C$ for any $n>0$.

Morita equivalence is the correct notion of equivalence for 0-cells in a bicategory. As a result it should respect the Euler characteristic and the trace. We now consider both of these, starting with the special case of the Euler characteristic.

Proposition 4.6. Suppose $(M, N)$ is a Morita equivalence. Then $\chi_{(M, N)}(M)=\chi_{(M, N)}(N)$ is an isomorphism with inverse $\chi_{(N, M)}(N)=\chi_{(N, M)}(M)$.

Proof. Figure 4.7a is a graphical representation of the composite of Euler characteristics where we have nested the red/green dual pair inside the orange/blue dual pair. Following Figure 4.4 we can first cancel the concentric green and orange regions resulting in Figure 4.7b. Then the red and blue regions cancel. 
the commutative diagrams below are a more formal proof.

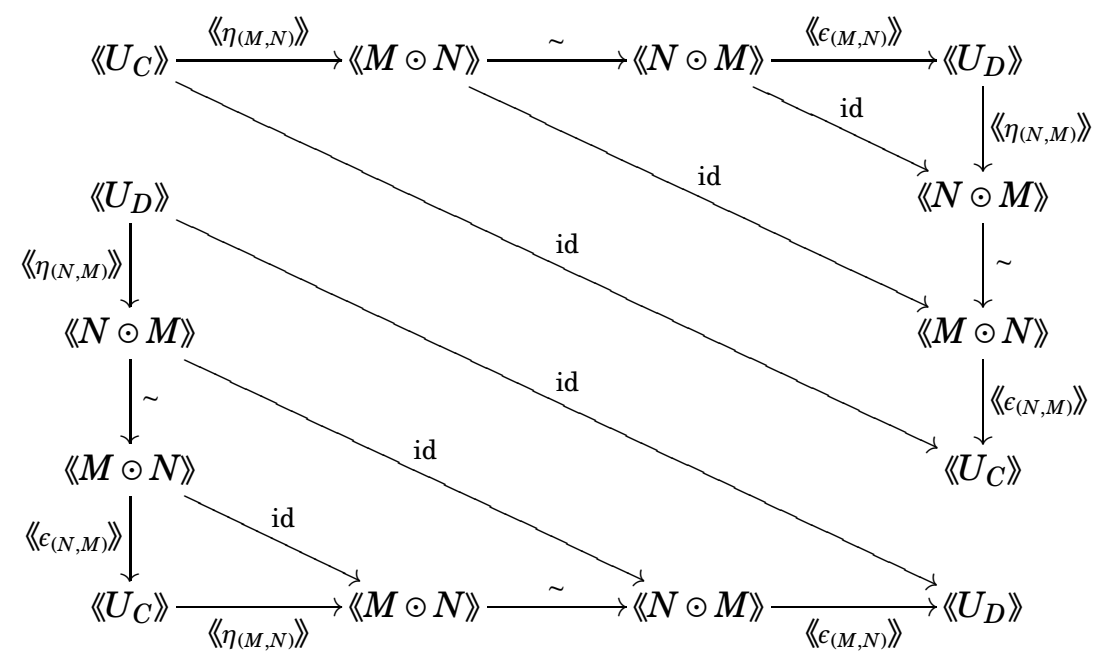

More generally, we have the following proposition.

Proposition 4.8. Suppose $(M, N)$ is a Morita equivalence. If $Q$ is a 1-cell so that $N \odot Q \odot$ $M$ is defined,

$$
\langle Q\rangle \stackrel{\operatorname{tr}\left(\eta_{Q}^{(M, N)}\right)}{\longrightarrow}\langle N \odot Q \odot M\rangle
$$

is an isomorphism with inverse $\left\langle\langle N \odot Q \odot M\rangle \stackrel{\operatorname{tr}\left(\epsilon_{Q}^{(N, M)}\right)}{\longrightarrow}\langle Q\rangle\right\rangle$.

Proof. Recall from Lemma 3.13 that the trace of $\epsilon_{Q}^{(N, M)}$ is

$$
\langle N \odot Q \odot M\rangle \stackrel{\sim}{\rightarrow}\langle Q \odot M \odot N\rangle \stackrel{\left\langle\mathrm{id}_{Q} \odot \epsilon_{(N, M)}\right\rangle}{\longrightarrow}\left\langle Q Q \odot U_{C}\right\rangle \cong\langle Q\rangle
$$

and the trace of $\eta_{Q}^{(M, N)}$ is

$$
\langle Q\rangle \cong\left\langle Q Q \odot U_{C}\right\rangle \stackrel{\left\langle\mathrm{id}_{Q} \odot \eta\right\rangle}{\longrightarrow}\langle Q \odot \odot M \odot N\rangle \cong\langle\langle N \odot Q \odot M\rangle .
$$

Composing these maps in both orders we have the following commutative diagrams.

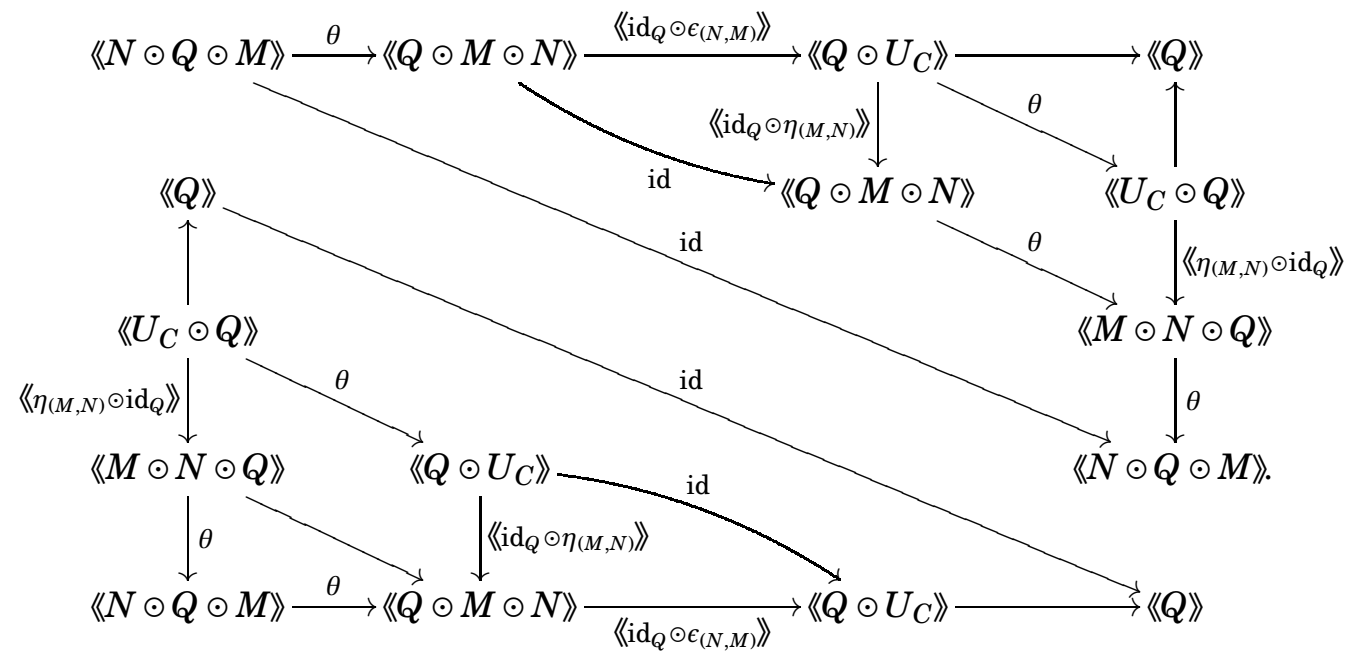


Corollary 4.9. Suppose $\left(M_{1}, N_{1}\right)$ is a dual pair, $\left(M_{2}, N_{2}\right)$ is a Morita equivalence, $Q$ and $P$ are 1-cells so that $M_{1} \odot Q \odot N_{1}$ and $N_{2} \odot P \odot M_{2}$ are defined. For a right dualizable 1cell $L$ so that $M_{1} \odot L \odot M_{2}$ is defined, and a 2-cell $f: Q \odot L \rightarrow L \odot P$ the following diagram commutes.

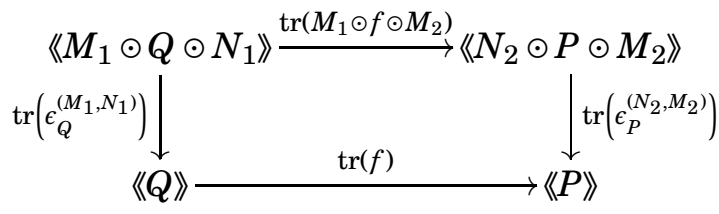

Proof. This follows from Proposition 4.8 and Corollary 3.20.

\section{EUlER CHARACTERISTICS FOR BASE CHANGE OBJECTS}

Given a map $f: A \rightarrow C$ of monoids in a symmetric monoidal category $\mathscr{V}$, we can define an $(A, C)$-bimodule ${ }_{f} C$ which has an action on the left through $f: A \rightarrow C$. Similarly, we get a $(C, A)$-bimodule $C_{f}$. These objects are used to change base in the following sense. Given a $(C, D)$-bimodule $M$, computing the composition ${ }_{f} C \odot M$ is the same as computing $\left(f \times \mathrm{id}_{D}\right)^{*} M$. These types of objects arise in any bicategory defined from an indexed monoidal category [PS12], but we need not work in that generality here. Instead, we work in the two examples from Section 3.

The main concern of $\$ 5.1$ will be recovering the classical "Morita invariance" statement that $\mathrm{THH}(A) \stackrel{\sim}{\rightarrow} \mathrm{THH}\left(\operatorname{Mod}_{A}^{c}\right)$ as well as a new, twisted, version of that statement by working in bicategories. Unraveling the necessary category theory, this hinges on the following question. Given a functor of $\mathscr{V}$-categories $F: \mathscr{A} \rightarrow \mathscr{C}$, we have an associated map of 2-cells $F: U_{\mathscr{A}} \rightarrow U_{\mathscr{C}}$. Applying the shadow, we get a map $\left\langle U_{\mathscr{A}}\right\rangle \rightarrow\left\langle\left\langle U_{\mathscr{C}}\right\rangle\right.$. We could instead consider the 1-cell $F_{\mathscr{C}} \mathscr{C}$ (defined carefully in Definition 5.3) and compute $\chi\left({ }_{F} \mathscr{C}\right):\left\langle U_{\mathscr{A}}\right\rangle \rightarrow\left\langle\left\langle U_{\mathscr{C}}\right\rangle\right\rangle$. It is clear that these maps should be the same, and we verify this in Lemma 5.8. This simple observation is the core of what allows us to prove our main theorems.

In $§ 5.2$ we use this identification to describe traces across Morita equivalences. Combining Theorem 5.14 and Lemma 5.8 gives Corollary 5.20. This result is then used to show Theorem 1.1.

5.1. Morita equivalence arising from base change. In what follows, let $\mathscr{V}$ be a symmetric monoidal category.

Remark 5.1. We may choose $\mathscr{V}$ to be any symmetric monoidal model category that satisfies the conditions of [SS03, Prop. 6.1]. In that case, the categories described below have associated model structures and homotopy categories.

Definition 5.2. Let $f: A \rightarrow C$ be a morphism in $\operatorname{Mon}(\mathscr{V})$. Then there is an $(A, C)$ bimodule ${ }_{f} C$, which is $C$ with a left $A$-action given by $f$, and a $(C, A)$-bimodule $C_{f}$ which is $C$ with a right $A$-action given by $f$.

Definition 5.3. Let $F: \mathscr{A} \rightarrow \mathscr{C}$ be a morphism in $\operatorname{Cat}(\mathscr{V})$, i.e. a functor of categories enriched in $\mathscr{V}$. Then there is an $(\mathscr{A}, \mathscr{C})$-bimodule ${ }_{F} \mathscr{C}$ where the left action of $\mathscr{A}$ is given by

$$
\begin{aligned}
\mathscr{A}\left(a, a^{\prime}\right) \otimes\left({ }_{F} \mathscr{C}\right)\left(a^{\prime}, c\right) & =\mathscr{A}\left(a, a^{\prime}\right) \otimes \mathscr{C}\left(F\left(a^{\prime}\right), c\right) \\
& \rightarrow \mathscr{C}\left(F(a), F\left(a^{\prime}\right)\right) \otimes \mathscr{C}\left(F\left(a^{\prime}\right), c\right) \rightarrow \mathscr{C}(F(a), c)={ }_{F} \mathscr{C}(a, c)
\end{aligned}
$$

There is a dual $(\mathscr{C}, \mathscr{A})$-bimodule that we denote $\mathscr{C}_{F}$.

Definition 5.4. We call any of ${ }_{f} C, C_{f},{ }_{F} \mathscr{C}, \mathscr{C}_{F}$ base change 1-cells. 
Remark 5.5. It is important to remember that the maps $f: A \rightarrow C$ and $F: \mathscr{A} \rightarrow \mathscr{C}$ are not 1-cells in the categories $\mathscr{B}(\operatorname{Mon}(\mathscr{V}))$ and $\mathscr{B}(\mathbf{C a t}(\mathscr{V}))$. They are the vertical 1-cells in an attendant double category (see, e.g. [Shu08]).

In special cases, base change one cells may exhibit a Morita equivalence. We isolate this special case in a definition.

Definition 5.6. A $\mathscr{V}$-functor $F: \mathscr{A} \rightarrow \mathscr{C}$ is a Morita equivalence if $\left({ }_{F} \mathscr{C}, \mathscr{C}_{F}\right)$ is a Morita equivalence. In particular, $F$ is a Morita equivalence if and only if $F$ is full and faithful and the map induced by composition

$$
\mathscr{C}(c, F(-)) \odot \mathscr{C}\left(F(-), c^{\prime}\right) \rightarrow \mathscr{C}\left(c, c^{\prime}\right)
$$

is an isomorphism.

The first step in answering the questions posed in the introduction to this section is to give descriptions of the coevaluation, evaluation, and Euler characteristic for base change objects.

Proposition 5.7. [Pon10, Appendix][PS12, Lem. 7.6]

i. If $f: A \rightarrow C$ is a monoid homomorphism, $\left({ }_{f} C, C_{f}\right)$ is a dual pair.

ii. If $F: \mathscr{A} \rightarrow \mathscr{C}$ be a $\mathscr{V}$-functor between $\mathscr{V}$-categories, $\left({ }_{F} \mathscr{C}, \mathscr{C}_{F}\right)$ is a dual pair.

For objects $a$ and $a^{\prime}$ in $\mathscr{A}$, a choice for the coevaluation is

$$
\begin{aligned}
\mathscr{A}\left(a, a^{\prime}\right) \stackrel{F}{\rightarrow} \mathscr{C}\left(F(a), F\left(a^{\prime}\right)\right) & \cong \mathscr{C}\left(F(a), F\left(a^{\prime}\right)\right) \otimes 1_{\mathscr{V}} \\
& \rightarrow \mathscr{C}\left(F(a), F\left(a^{\prime}\right)\right) \otimes \mathscr{C}\left(F\left(a^{\prime}\right), F\left(a^{\prime}\right)\right) \rightarrow \mathscr{C}(F(a),-) \odot \mathscr{C}\left(-, F\left(a^{\prime}\right)\right)
\end{aligned}
$$

For objects $c, c^{\prime}$ of $\mathscr{C}$, the corresponding evaluation is induced by the composition of morphisms as in the following diagram.

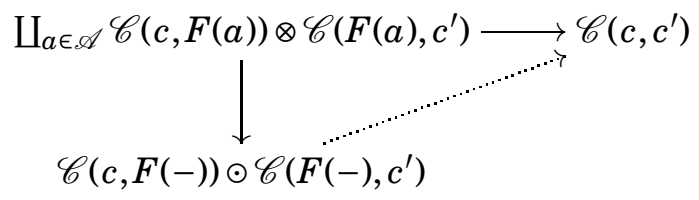

If $\mathscr{M}$ is a $(\mathscr{C}, \mathscr{D})$-bimodule and $F: \mathscr{A} \rightarrow \mathscr{C}$ and $G: \mathscr{B} \rightarrow \mathscr{D}$ are enriched functors, $F \mathscr{M}_{G}$ is the $(\mathscr{A}, \mathscr{B})$-bimodule defined as the composite

$$
\mathscr{A}^{\mathrm{op}} \otimes \mathscr{B} \stackrel{F \otimes G}{\longrightarrow} \mathscr{C}^{\mathrm{op}} \otimes \mathscr{D} \stackrel{\mathscr{M}}{\longrightarrow} \mathscr{V}
$$

If $\mathscr{Q}$ is an $(\mathscr{C}, \mathscr{C})$-bimodule and $F$ is as above, we have the following composite

$$
\epsilon_{\mathscr{Q}}^{F}:{ }_{F} \mathscr{Q}_{F} \odot{ }_{F} \mathscr{C} \cong{ }_{F} \mathscr{C} \odot \mathscr{Q} \odot \mathscr{C}_{F} \odot{ }_{F} \mathscr{C} \stackrel{\epsilon_{\mathscr{Q}}^{\left(\mathscr{C}_{,} \mathscr{C}_{F}\right)}}{\longrightarrow}{ }_{F} \mathscr{C} \odot \mathscr{Q}
$$

and the corresponding maps for $\eta_{\mathscr{Q}}^{F},{ }^{F} \epsilon_{\mathscr{Q}}$, and ${ }^{F} \eta_{\mathscr{Q}}$. The are also versions of these maps for monoids.

The following statement (and its restriction to the case of monoids) follows immediately from the coevaluation and evaluation above.

Lemma 5.8. Let $F: \mathscr{A} \rightarrow \mathscr{C}$ be a $\mathscr{V}$-functor between $\mathscr{V}$-categories and $\mathscr{Q}$ be a $(\mathscr{C}, \mathscr{C})$ bimodule. Then the following two diagrams commute.
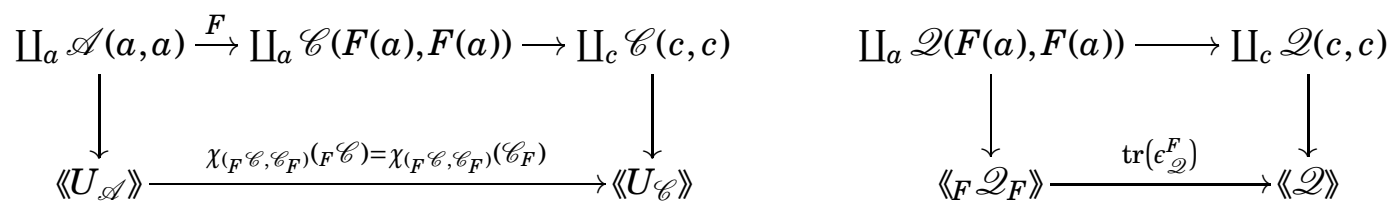
While unassuming and an immediate consequence of this choice of evaluation and coevaluation for base change dual pairs, this lemma is a fundamental connection between traces and maps of hom sets. The left diagram above implies

$$
\chi_{\left(F, \mathscr{C}, \mathscr{C}_{F}\right)}\left({ }_{F} \mathscr{C}\right)=\chi_{\left({ }_{F} \mathscr{C}, \mathscr{C}_{F}\right)}\left(\mathscr{C}_{F}\right)=\langle\langle F\rangle .
$$

This observation will be used in Corollaries 5.9, 5.16 and 5.20. If $F$ is the inclusion of a subcategory, $\operatorname{tr}\left(\epsilon_{\mathscr{Q}}^{F}\right)$ is the map on shadows induced by that inclusion.

Corollary 5.9. If $F: \mathscr{A} \rightarrow \mathscr{C}$ is a Morita equivalence and $\mathscr{Q}$ is a $(\mathscr{C}, \mathscr{C})$-bimodule

$$
\langle F\rangle\rangle:\langle\mathscr{A}\rangle\rangle \rightarrow\langle\mathscr{C}\rangle \quad \text { and } \operatorname{tr}\left(\epsilon_{\mathscr{Q}}^{F}\right):\left\langle\left\langle_{F} \mathscr{Q}_{F}\right\rangle \rightarrow\langle\langle\mathscr{Q}\rangle\right.
$$

are isomorphisms.

Proof. Propositions 4.6 and 4.8 with the substitutions

$$
\begin{array}{c|c|c}
M & N & Q \\
\hline F^{\mathscr{C}} & \mathscr{C}_{F} & \mathscr{Q}
\end{array}
$$

imply

$$
\chi_{\left(F, \mathscr{C}_{F}\right)}\left({ }_{F} \mathscr{C}\right)=\chi_{\left({ }_{F} \mathscr{C}, \mathscr{C}_{F}\right)}\left(\mathscr{C}_{F}\right):\langle\mathscr{A}\rangle \rightarrow\langle\mathscr{C}\rangle \quad \text { and } \operatorname{tr}\left(\epsilon_{\mathscr{Q}}^{F}\right):\left\langle\left\langle_{F} \mathscr{Q}_{F}\right\rangle \rightarrow\langle\langle\mathscr{Q}\rangle\right.
$$

are isomorphisms. The remaining identification follows from Lemma 5.8.

The following is a crucial example. It is a classical fact for rings [DM96, Prop. 2.1.5], and known for spectra [BM12] and it provides important motivation for this paper. The example shows that it follows from purely bicategorical facts.

Example 5.10. Let $\mathscr{B}(\mathbf{C a t}(\mathrm{Sp}))$ be the bicategory of spectral categories (with the proper homotopy theoretic considerations, see Example 3.1). Let $A$ be a ring spectrum. There are two spectral categories naturally associated with $A$

$i$. The spectral category $\operatorname{Mod}_{A}^{c}$.

$i i$. The one object spectral category whose hom spectrum is $A$. We denote this category $\operatorname{End}_{\operatorname{Mod}_{A}^{c}}(A)$ since it is the full subcategory of $\operatorname{Mod}_{A}^{c}$ with the single object A.

There is an inclusion functor

$$
E_{A}: \operatorname{End}_{\operatorname{Mod}_{A}^{c}}(A) \rightarrow \operatorname{Mod}_{A}^{c}
$$

and so we can construct a 1-cell (i.e. a spectral bimodule) $E_{A}\left(\operatorname{Mod}_{A}^{c}\right)$. There is similarly a spectral bimodule $\left(\operatorname{Mod}_{A}^{c}\right)_{E_{A}}$. These two bimodules are a Morita equivalence.

Applying Corollary 5.9 with the substitutions

the map

\begin{tabular}{c|c|c}
$\mathscr{A}$ & $\mathscr{C}$ & $F$ \\
\hline $\operatorname{End}_{\operatorname{Mod}_{A}^{c}}(A)$ & $\operatorname{Mod}_{A}^{c}$ & $E_{A}$
\end{tabular}

$$
\mathrm{THH}(A) \cong \mathrm{THH}\left(\operatorname{End}_{\operatorname{Mod}_{A}^{c}}(A)\right) \rightarrow \mathrm{THH}\left(\operatorname{Mod}_{A}^{c}\right)
$$

induced by the inclusion of $A$ into $\operatorname{Mod}_{A}^{c}$ as a module over itself is an isomorphism. We will give an explicit description of the inverse of this map on $\pi_{0}$ in Theorem 7.8.

Example 5.12. Let $A$ be a ring spectrum, $Q$ be an $(A, A)$-bimodule, and

$$
-\wedge_{A} Q: \operatorname{Mod}_{A}^{c} \rightarrow \operatorname{Mod}_{A}^{c}
$$

be given by $M \mapsto M \wedge_{A} Q$. Then $\left(\operatorname{Mod}_{A}^{c}\right)_{-\wedge_{A} Q}$ is an $\left(\operatorname{Mod}_{A}^{c}\right.$, $\left.\operatorname{Mod}_{A}^{c}\right)$-bimodule and the inclusion

$$
E_{A}: \operatorname{End}_{\operatorname{Mod}_{A}^{c}}(A) \rightarrow \operatorname{Mod}_{A}^{c}
$$


defines a $\left(\operatorname{End}_{\operatorname{Mod}_{A}^{c}}(A), \operatorname{End}_{\operatorname{Mod}_{A}^{c}}(A)\right)$-bimodule $E_{A}\left(\left(\operatorname{Mod}_{A}^{c}\right)_{-\wedge_{A} Q}\right)_{E_{A}}$.

Since $E_{A}$ is a Morita equivalence, applying Corollary 5.9 with the substitutions

\begin{tabular}{c|c|c|c}
$\mathscr{A}$ & $\mathscr{C}$ & $F$ & $\mathscr{Q}$ \\
\hline $\operatorname{End}_{\operatorname{Mod}_{A}^{c}(A)}(A) \operatorname{Mod}_{A}^{c}$ & $E_{A}$ & $\left(\operatorname{Mod}_{A}^{c}\right)_{-\wedge_{A} Q}$
\end{tabular}

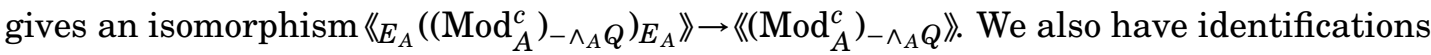

$$
\begin{aligned}
& \left\langle E_{A}\left(\left(\operatorname{Mod}_{A}^{c}\right)_{-\wedge_{A} Q}\right)_{E_{A}}\right\rangle=\langle\langle Q\rangle=\operatorname{THH}(A ; Q) \\
& \left\langle\left(\operatorname{Mod}_{A}^{c}\right)_{-\wedge_{A} Q} Q=\left\langle\left(\operatorname{Mod}_{A}^{c}\right)_{-\wedge_{A} Q}\right\rangle=\operatorname{THH}\left(\operatorname{Mod}_{A}^{c} ;-\wedge_{A} Q\right)\right.
\end{aligned}
$$

and so an isomorphism

$$
\operatorname{THH}(A ; Q) \rightarrow \operatorname{THH}\left(\operatorname{Mod}_{A}^{c} ;-\wedge_{A} Q\right)
$$

Thus, the classical Morita invariance of THH holds in a twisted context as well.

5.2. Morita equivalence and trace. We now turn to the comparison of traces across Morita equivalence following Propositions 4.6 and 4.8. We start with an example to help motivate the following results. If $\phi: M \rightarrow M$ is a endomorphism of a right dualizable $(C, D)$-bimodule the trace of $\phi$ is a map

$$
\mathrm{THH}(C) \rightarrow \mathrm{THH}(D) .
$$

We can also consider the functor $\operatorname{Mod}_{C}^{c} \rightarrow \operatorname{Mod}_{D}^{c}$ given by tensoring with $M$ on objects and tensoring with $\phi$ on morphisms. With the Morita equivalences for $C$ and $\operatorname{Mod}_{C}^{c}$ and for $D$ and $\operatorname{Mod}_{D}^{c}$ these give us the following diagram.

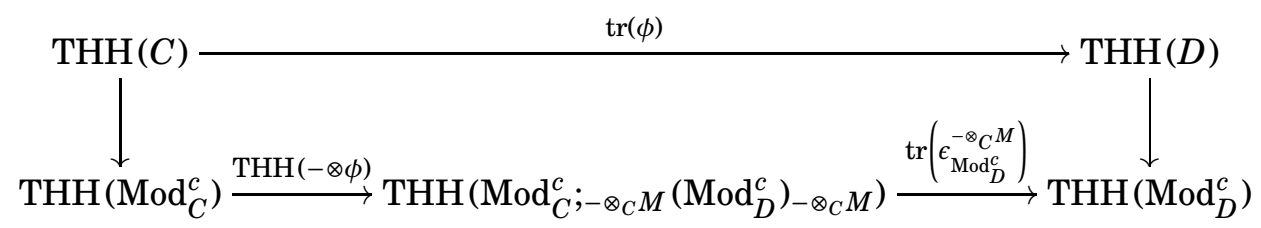

At this level of generality we can directly confirm this diagram commutes, but it will be more convenient to prove a significant generalization and then verify that this diagram is a special case. This generalization (Theorem 5.14) is one of the main results of the paper and underlies the ideas in Section 6.

We first fix some notation.

Definition 5.13. If $\mathscr{C}$ is a category enriched in $\mathscr{V}$ and $c$ is an object of $\mathscr{C}$ let $\operatorname{End}_{\mathscr{C}}(c)$ denote the full subcategory of $\mathscr{C}$ whose single object is $c$. Let $E_{c}: \operatorname{End}_{\mathscr{C}}(c) \rightarrow \mathscr{C}$ be the inclusion.

Theorem 5.14. Let $\mathscr{C}$ and $\mathscr{D}$ be categories enriched over $\mathscr{V}, c$ be an object of $\mathscr{C}, d$ be an object of $\mathscr{D}, \mathscr{M}$ be a right dualizable $(\mathscr{C}, \mathscr{D})$-bimodule, and suppose $E_{d}$ is a Morita equivalence. Let $\mathscr{Q}$ be a $(\mathscr{C}, \mathscr{C})$-bimodule, $\mathscr{P}$ be a $(\mathscr{D}, \mathscr{D})$-bimodule and

$$
\phi: \mathscr{Q} \odot \mathscr{M} \rightarrow \mathscr{M} \odot \mathscr{P}
$$

be a natural transformation. Then

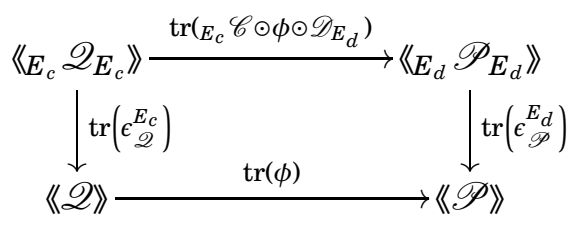

commutes.

Proof. This is a consequence of Corollary 4.9 with the substitutions 


\begin{tabular}{c|c|c|c|c|c|c|c}
$M_{1}$ & $N_{1}$ & $M_{2}$ & $N_{2}$ & $Q$ & $P$ & $L$ & $f$ \\
\hline$E_{c} \mathscr{C}$ & $\mathscr{C}_{E_{c}}$ & $\mathscr{D}_{E_{d}}$ & $E_{d} \mathscr{D}$ & $\mathscr{Q}$ & $\mathscr{P}$ & $\mathscr{M}$ & $\phi$
\end{tabular}

We can now recover a main result of Lind-Malkiewich [LM16, Prop. 5.5].

Corollary 5.16. Let $\mathscr{A}$ and $\mathscr{C}$ be categories enriched over $\mathscr{V}$, a be an object of $\mathscr{A}, c$ be an object of $\mathscr{C}$, and $F: \mathscr{A} \rightarrow \mathscr{C}$ be a $\mathscr{V}$-functor. If $E_{c}: \operatorname{End}_{\mathscr{C}}(c) \rightarrow \mathscr{C}$ is a Morita equivalence,

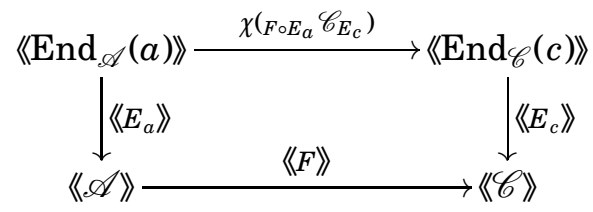

commutes.

Note that ${ }_{F \circ E_{a}} \mathscr{C}_{E_{c}}$ is $\mathscr{C}(F(a), c)$ is as a $\left(\operatorname{End}_{\mathscr{A}}(a), \operatorname{End}_{\mathscr{C}}(c)\right)$-bimodule.

Proof. This follows from Theorem 5.14 with the substitutions

\begin{tabular}{c|c|c|c|c|c|c|c}
$\mathscr{C}$ & $\mathscr{D}$ & $c$ & $d$ & $\mathscr{M}$ & $\mathscr{Q}$ & $\mathscr{P}$ & $\phi$ \\
\hline $\mathscr{A}$ & $\mathscr{C}$ & $a$ & $c$ & $F^{\mathscr{C}}$ & $U_{\mathscr{A}}$ & $U_{\mathscr{C}}$ & $\mathrm{id}$
\end{tabular}

Lemma 5.8 identifies the vertical and bottom maps.

Example 5.17. Let $C$ and $D$ be rings and $M$ be a $(C, D)$-bimodule that is that is finitely generated and projective as an right $D$-module.

By Corollary 5.16 with the substitutions

\begin{tabular}{c|c|c|c|c}
$\mathscr{A}$ & $a$ & $\mathscr{C}$ & $c$ & $F$ \\
\hline $\operatorname{Mod}_{C}^{c}$ & $C$ & $\operatorname{Mod}_{D}^{c}$ & $D$ & $-\otimes_{C} M$
\end{tabular}

following diagram, where the vertical maps are inclusions, commutes.

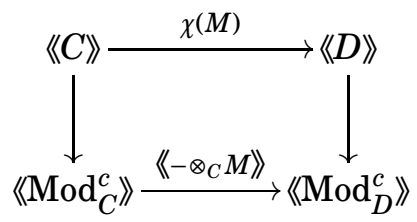

In later examples the map $\phi$ in Theorem 5.14 is a composite

$$
\mathscr{Q} \odot \mathscr{M} \stackrel{\psi \odot \mathrm{id}_{\mathscr{M}}}{\longrightarrow} \mathscr{M} \odot \mathscr{P} \odot \mathscr{N} \odot \mathscr{M} \stackrel{\epsilon_{\mathscr{P}}^{(\mathscr{M}, \mathscr{N})}}{\longrightarrow} \mathscr{M} \odot \mathscr{P}
$$

for a map $\psi: \mathscr{Q} \rightarrow \mathscr{M} \odot \mathscr{P} \odot \mathscr{N}$. Then Proposition 3.21 implies the trace of (5.18) is the composite $\langle\mathscr{Q}\rangle \stackrel{\| \psi\rangle\rangle}{\longrightarrow}\langle\mathscr{M} \odot \mathscr{P} \odot \mathscr{N}\rangle \stackrel{\operatorname{tr}\left(\epsilon_{\mathscr{P}}^{(\mathscr{M}, N)}\right)}{\longrightarrow}\langle\mathscr{P}\rangle$ and (5.15) becomes

$$
\begin{aligned}
& \left\langle E_{c} \mathscr{Q}_{E_{c}}\right\rangle \longrightarrow \quad \operatorname{tr}\left(E_{c} \mathscr{C} \odot \phi \odot \mathscr{D}_{E_{d}}\right) \longrightarrow\left\langle E_{d} \mathscr{P}_{E_{d}}\right\rangle
\end{aligned}
$$

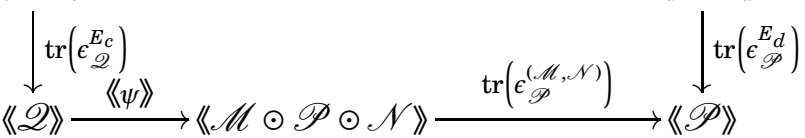

An important example of this is the 2-cells that arise from a natural transformation as in Corollary 5.20. We first describe how these two cells are defined. For enriched categories $\mathscr{A}$ and $\mathscr{C}$, enriched functors

$$
J: \mathscr{A} \rightarrow \mathscr{A}, K: \mathscr{C} \rightarrow \mathscr{C} \text { and } F: \mathscr{A} \rightarrow \mathscr{C},
$$


and a natural transformation $\Phi: F \circ J \rightarrow K \circ F$ let $\phi: \mathscr{A}_{J} \odot_{F} \mathscr{C} \rightarrow{ }_{F} \mathscr{C} \odot \mathscr{C}_{K}$ be the 2-cell defined by

$$
\left(a \stackrel{\alpha}{\rightarrow} J\left(a^{\prime}\right), F\left(a^{\prime}\right) \stackrel{\beta}{\rightarrow} c\right) \mapsto\left(F(a) \stackrel{F(\alpha)}{\longrightarrow} F\left(J\left(a^{\prime}\right)\right) \stackrel{\Phi_{a^{\prime}}}{\longrightarrow} K\left(F\left(a^{\prime}\right)\right) \stackrel{K(\beta)}{\longrightarrow} K(c)\right)
$$

and $\psi: \mathscr{A}_{J} \rightarrow{ }_{F} \mathscr{C} \odot \mathscr{C}_{K} \odot \mathscr{C}_{F}$ be 2-cell defined by

$$
\left(a \stackrel{\alpha}{\rightarrow} J\left(a^{\prime}\right)\right) \mapsto\left(F(a) \stackrel{F(\alpha)}{\longrightarrow} F\left(J\left(a^{\prime}\right)\right) \stackrel{\Phi_{a^{\prime}}}{\longrightarrow} K\left(F\left(a^{\prime}\right)\right)\right)
$$

This choice of $\phi$ and $\psi$ are related as in (5.18).

Corollary 5.20. For $\mathscr{A}, \mathscr{C}, J, K, F, \phi$ and $\psi$ as above and objects $a$ of $\mathscr{A}$ and $c$ of $\mathscr{C}$, the following diagrams, where vertical maps are induced by inclusions on hom sets, commute.

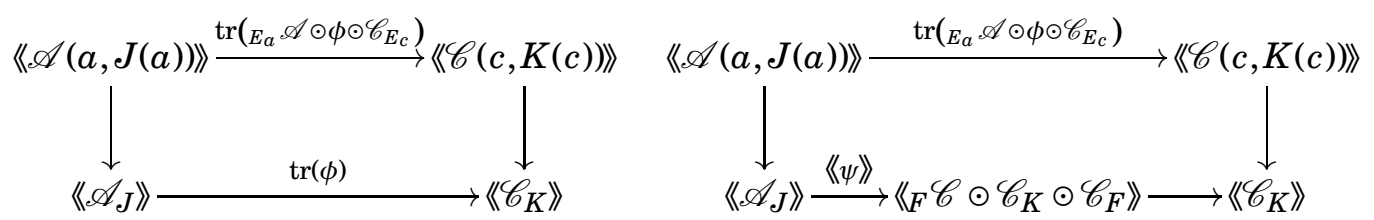

The remaining unlabeled map is induced by the map

$$
\coprod_{a \in \mathscr{A}} \mathscr{C}(F(a), K(F(a))) \rightarrow \coprod_{c \in \mathscr{C}} \mathscr{C}(c, K(c)) .
$$

Proof. Theorem 5.14 with the substitutions

\begin{tabular}{c|c|c|c|c|c|c}
$\mathscr{C}$ & $c$ & $\mathscr{D}$ & $d$ & $\mathscr{M}$ & $\mathscr{Q}$ & $\mathscr{P}$ \\
\hline $\mathscr{A}$ & $a$ & $\mathscr{C}$ & $c$ & $F^{\mathscr{C}}$ & $\mathscr{A}_{J}$ & $\mathscr{C}_{K}$
\end{tabular}

gives the following commutative diagram.

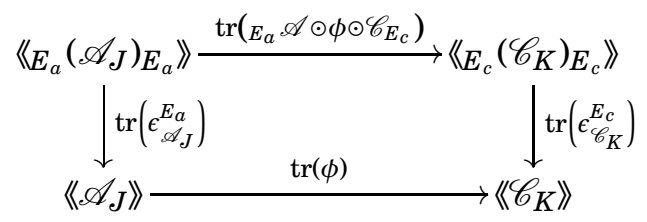

The diagram in (5.19) becomes

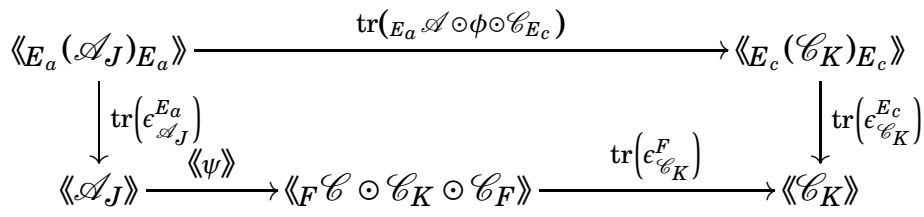

The remaining simplifications follow from Lemma 5.8.

For later applications it is convenient to note that $E_{a} \mathscr{A} \odot \phi \odot \mathscr{C}_{E_{c}}$ is

$$
\begin{aligned}
\mathscr{A}(a, J(a)) \odot \mathscr{C}(F(a), c) & \rightarrow \mathscr{C}(F(a), K(c)) \\
\quad(a \stackrel{\alpha}{\longrightarrow} J(a), F(a) \stackrel{\beta}{\rightarrow} c) & \mapsto\left(F(a) \stackrel{F(\alpha)}{\longrightarrow} F(J(a)) \stackrel{\Phi_{a}}{\longrightarrow} K(F(a)) \stackrel{K(\beta)}{\longrightarrow} K(c)\right)
\end{aligned}
$$

Example 5.21. We now return to the example at the beginning of this subsection. Let $C$ and $D$ be rings, $Q$ be an $(C, C)$-bimodule, $P$ be an $(D, D)$-bimodule, $M$ be an $(C, D)$ bimodule that is finitely generated and projective as an right $D$-module, and let $f: Q \otimes_{C}$ $M \rightarrow M \otimes_{D} P$ be a homomorphism.

Corollary 5.20 with the substitutions

\begin{tabular}{c|c|c|c|c|c|c|c}
$\mathscr{A}$ & $a$ & $\mathscr{C}$ & $c$ & $J$ & $K$ & $F$ & $\Phi$ \\
\hline $\operatorname{Mod}_{C}^{c}$ & $C$ & $\operatorname{Mod}_{D}^{c}$ & $D$ & $-\otimes_{C} Q$ & $-\otimes_{D} P$ & $-\otimes_{C} M$ & $-\otimes_{C} f$
\end{tabular}


implies the bottom square in the following diagram commutes.

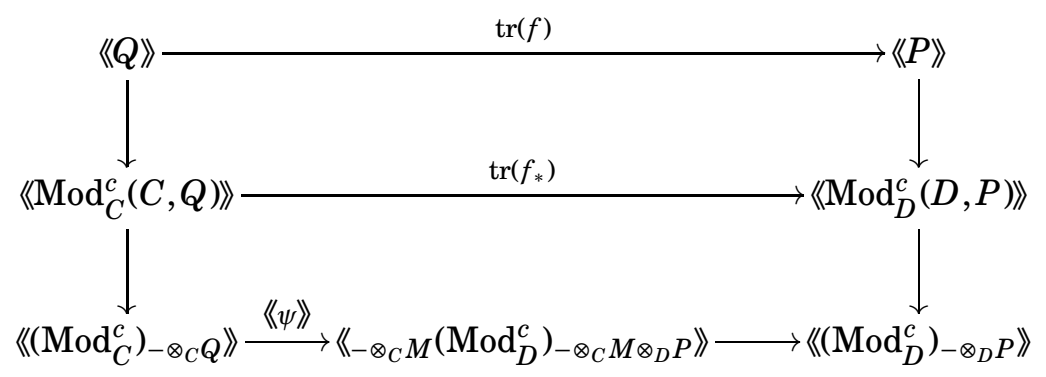

In this example, $E_{C}\left(\operatorname{Mod}_{C}^{c}\right) \odot \phi \odot\left(\operatorname{Mod}_{D}^{c}\right)_{E_{D}}$ is

$$
\begin{aligned}
\operatorname{Mod}_{C}^{c}(C, Q) \odot \operatorname{Mod}_{D}^{c}(M, D) & \rightarrow \operatorname{Mod}_{D}^{c}(M, P) \\
(C \stackrel{\alpha}{\rightarrow} Q, M \stackrel{\beta}{\rightarrow} D) & \mapsto\left(C \otimes_{C} M \stackrel{\alpha \otimes \mathrm{id}}{\longrightarrow} Q \otimes_{A} M \stackrel{f}{\rightarrow} M \otimes_{D} P \stackrel{\beta \otimes \mathrm{id}}{\longrightarrow} D \otimes_{D} P\right)
\end{aligned}
$$

The top square is the identification of $\operatorname{Mod}_{C}^{c}(C, Q)$ with $Q$ and $\operatorname{Mod}_{D}^{c}(D, P)$ with $P$ and the observation that the trace of the dual of a map is the trace of the map.

\section{EXAMPLe: Fixed Point InVARIANTS}

We now return to the motivating example from fixed point theory described in the introduction and prove Theorem 1.1 in Theorem 6.10.

Let $R$ be a ring spectrum and $A, C$ be $R$-algebras. A map of $R$-algebras $f: A \rightarrow C$ defines an $(A, C)$-bimodule ${ }_{f} C$. If ${ }_{f} C$ is left dualizable, we have an adjunction

$$
C_{f} \wedge_{A}-: \operatorname{Mod}_{(A, R)}^{l c} \leftrightarrows \operatorname{Mod}_{(C, R)}^{l c}:{ }_{f} C \wedge_{C}-
$$

between the bimodules that are left-compact (that is, for example, an $(A, R)$-bimodule is compact when considered as an $A$-module). Letting $R$ be the sphere spectrum and taking THH of both sides of the adjunction gives us maps

$$
\text { res: } \mathrm{THH}\left(\operatorname{Mod}_{A}^{c}\right) \leftrightarrows \mathrm{THH}\left(\operatorname{Mod}_{C}^{c}\right): \operatorname{trf}
$$

which are usually referred to as restriction and transfer. There is a restriction for any map $f$, but there is only a transfer if $C$ is compact as an $A$-module. Using Morita invariance restriction and transfer give maps between $\mathrm{THH}(A)$ and $\mathrm{THH}(C)$. We should note that the transfer map has appeared in many THH calculations and seems to provide powerful characteristic-type invariants [Sch98, Sch06, BM90].

It is well know that the composite

$$
S \cong \mathrm{THH}(S) \stackrel{\operatorname{trf}}{\longrightarrow} \mathrm{THH}\left(\Sigma_{+}^{\infty} \Omega X\right) \stackrel{\text { res }}{\longrightarrow} \mathrm{THH}(S) \simeq S
$$

is the Euler characteristic. In this section we show that similar results hold for the generalizations of the Euler characteristics used in fixed point theory. In particular we show that the spectrum-level Reidemeister trace of the second author [Pon10, Pon16] arises from transfer maps in THH. The transfer maps we use are "twisted" by a bimodule coordinate in THH.

Since transfer maps are nothing more than an example of base change we can apply the results of the previous section. Taking a more bicategorical perspective, we have a map

$$
U_{C} \odot C_{f} \rightarrow C_{f} \odot U_{A}
$$

which, upon taking traces gives us a $\operatorname{map}\left\langle\left\langle U_{C}\right\rangle \rightarrow\left\langle U_{A}\right\rangle\right.$, or $\operatorname{THH}(C) \rightarrow \operatorname{THH}(A)$. This is an example of what is referred to as an Euler characteristic above. Similarly,

$$
U_{A} \odot{ }_{f} C \rightarrow{ }_{f} C \odot U_{C}
$$


gives $\left\langle U_{A}\right\rangle \rightarrow\left\langle U_{C}\right\rangle$, i.e. $\mathrm{THH}(A) \rightarrow \mathrm{THH}(C)$. Then the following result is simply a restatement of Example 5.17.

Proposition 6.3. The diagrams
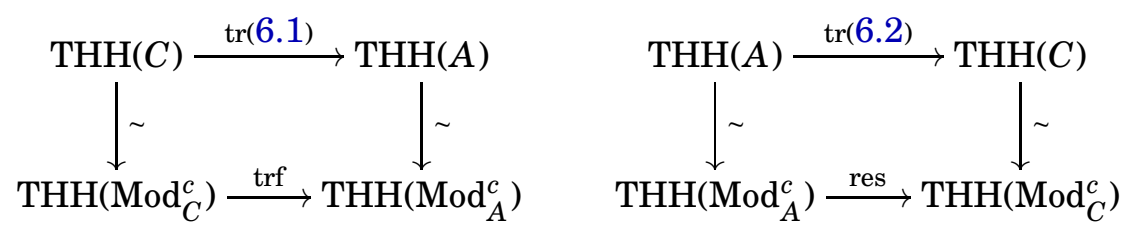

commute.

Remark 6.4. The above was proved in [LM16], as a step in verifying the first-named author's conjecture that the Becker-Gottlieb transfer factors through the THH transfer.

Thus, restriction and transfer maps are examples of a rather trivial bicategorical trace: the Euler characteristic. It is no coincidence that transfer maps in THH seem to produce results reminiscent of characteristics. The proposition further gives a very small, relatively computable model for the transfer map. In fact, it gives compact formulas for the usual restriction-transfer compositions

Theorem 6.5 (Restriction-Transfer).

$$
\begin{array}{r}
\text { resotrf }=\chi\left({ }_{f} C_{f}\right): \operatorname{THH}(A) \rightarrow \operatorname{THH}(A) \\
\text { trfores }=\chi\left(\left(C_{f}\right) \odot\left({ }_{f} C\right)\right): \operatorname{THH}(C) \rightarrow \operatorname{THH}(C) .
\end{array}
$$

Example 6.6. We present two examples of these types of restriction-transfer identities

- Let $X$ be a path-connected space of the homotopy type of a finite CW-complex. If we let $A=S$ and $C=\Sigma_{+}^{\infty} \Omega X$, then $A$ is compact as a $C$-module. The composite

$$
S \simeq \mathrm{THH}(S) \stackrel{\operatorname{trf}}{\longrightarrow} \mathrm{THH}\left(\Sigma_{+}^{\infty} \Omega X\right) \stackrel{\text { res }}{\longrightarrow} \mathrm{THH}(S) \simeq S
$$

is $\chi(X)$, regarded as an element of $\pi_{0}(S)$.

- Let $H$ be a subgroup of $G$ where $[G: H]<\infty$. Then the base change object associated to the inclusion of the group ring $A=\mathbb{Z}[H]$ into $C=\mathbb{Z}[G]$ is dualizable. The shadow is $\mathrm{HH}_{0}(\mathbb{Z}[G], \mathbb{Z}[G])$ and

$$
\mathrm{HH}_{0}(\mathbb{Z}[H], \mathbb{Z}[H]) \stackrel{\operatorname{trf}}{\longrightarrow} \mathrm{HH}_{0}(\mathbb{Z}[G], \mathbb{Z}[G]) \stackrel{\text { res }}{\longrightarrow} \mathrm{HH}_{0}(\mathbb{Z}[H], \mathbb{Z}[H])
$$

is multiplication by $[G: H]$. This has an interpretation in terms of the character theory of group representations, since $\mathrm{HH}_{0}(\mathbb{Z}[G], \mathbb{Z}[G]) \cong \mathbb{Z}\left[C_{G}\right]$ where $C_{G}$ denotes the conjugacy classes of $G$. Since maps from $\mathbb{Z}\left[C_{G}\right]$ are exactly class functions, this is the same $[G: H]$ that appears in the classical induction-restriction maps in character theory for representations of finite groups.

To extend these ideas to the Reidemeister trace we need an elaboration of the transfer. For algebras $A$ and $C$, a commuting diagram of maps of algebra

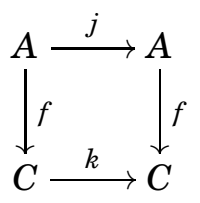


defines a commuting diagram of functors

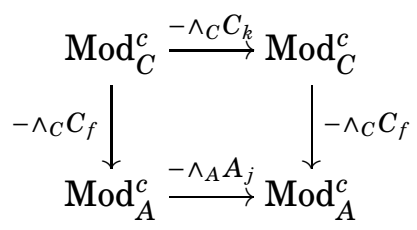

and induces maps

$$
\begin{aligned}
& C_{k} \odot C_{f} \rightarrow C_{f} \odot A_{j} .
\end{aligned}
$$

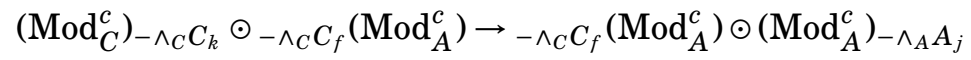

Proposition 6.9. Suppose $f, j$, and $k$ are as above. If $C_{f}$ is right dualizable then the following diagram commutes.

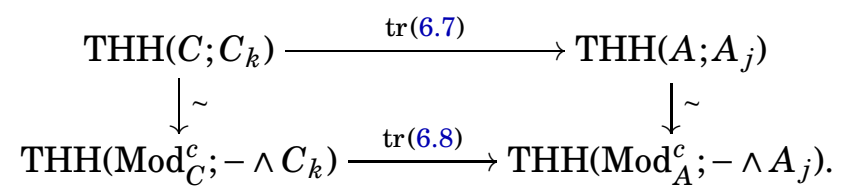

Proof. This follows from Corollary 5.20 with the substitutions

\begin{tabular}{c|c|c|c|c|c}
$\mathscr{A}$ & $\mathscr{C}$ & $I$ & $K$ & $F$ & $\Phi$ \\
\hline $\operatorname{Mod}_{C}^{c}$ & $\operatorname{Mod}_{A}^{c}$ & $-\wedge_{C} C_{k}$ & $-\wedge_{A} A_{j}$ & $-\wedge_{C} C_{f}$ & id
\end{tabular}

If $k$ is the identity map this reduced to a commutative diagram

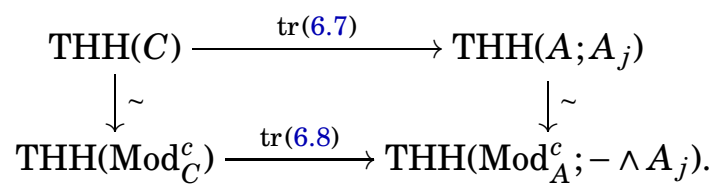

In this case the bottom map is the composite

$$
\mathrm{THH}\left(\operatorname{Mod}_{C}^{c}\right) \rightarrow \mathrm{THH}\left(\operatorname{Mod}_{A}^{c}\right) \rightarrow \mathrm{THH}\left(\operatorname{Mod}_{A}^{c} ;-\wedge A_{j}\right)
$$

where the first map is the transfer and the second map is induced by the map $S \rightarrow A_{j}$.

We now come to one of our main applications, which is simply a particular case of Proposition 6.9 (and so a consequence of Theorem 5.14). Let $X$ be path-connected space that has the homotopy type of a finite CW-complex, and let $f: X \rightarrow X$ be a self map. The map $X \rightarrow *$ gives a map $\Sigma_{+}^{\infty} \Omega X \rightarrow S$ which exhibits $S$ as a compact $\Sigma_{+}^{\infty} \Omega X$-module, and so there is a transfer $S \rightarrow \operatorname{THH}\left(\Sigma_{+}^{\infty} \Omega X\right)$. Also, $f$ induces a self-map $f: \Sigma_{+}^{\infty} \Omega X \rightarrow \Sigma_{+}^{\infty} \Omega X$.

Theorem 6.10. The Reidemeister trace of $f: X \rightarrow X$ is the THH transfer

$$
S \simeq \mathrm{THH}(S) \rightarrow \operatorname{THH}\left(\Sigma_{+}^{\infty} \Omega X ;-\wedge \Sigma_{+}^{\infty} \Omega X_{f}\right) \simeq \Sigma_{+}^{\infty} \mathscr{L} X^{f}
$$

Recall that twisted THH was defined in Definition 2.9 , and $\mathscr{L} X^{f}$ in the introduction — but see also Definition A.3.

Proof. In [Pon10, Prop. 3.2.3] the Reidemeister trace of the introduction is identified with a bicategorical trace. This trace is identified with a more homotopically satisfying trace in [Pon10, Prop. 6.2.2] and finally identified with a trace in the parameterized stable homotopy bicategory, Ex, in [Pon16, Thm. 4.1].

In the bicategory of parameterized spectra, let $S_{X}$ denote the fiberwise suspension spectrum of $X$ regarded as a space over $* \times X$ via the identity map. If $f: X \rightarrow X$ is a continuous map $X_{f}$ is the fiberwise suspension spectrum of $\left\{(\gamma, x) \in X^{I} \times X \mid \gamma(1)=f(x)\right\}$ 
regarded as a space over $X \times X$ via the map $(\gamma, x) \mapsto(\gamma(0), x)$. Then $f$ defines a map of spectra

$$
S \odot S_{X} \rightarrow S_{X} \odot X_{f}
$$

If $X$ is a closed smooth manifold, $S_{X}$ is right dualizable and the trace of (6.11) is a map $\left\langle\langle S\rangle \rightarrow\left\langle\left\langle X_{f}\right\rangle\right.\right.$. This map is one form of the Reidemeister trace.

Now, we use the Morita equivalence of [LM17] to compare the shadows in $\operatorname{Ex}(X, X)$ and $\left(\Sigma_{+}^{\infty} \Omega X, \Sigma_{+}^{\infty} \Omega X\right)$-bimodules. Let $\mathscr{B} \operatorname{imod}_{S}$ be the bicategory of ring spectra and spectral bimodules, and $\mathscr{B} \operatorname{imod}_{S}^{\text {gp }}$ be the full-subcategory of ring spectra of the form $\Sigma_{+}^{\infty} \Omega X$. Then, [LM17, Thm. 6.4] as applied in [LM16, Thm. 5.1] gives that the parameterized stable homotopy bicategory (when restricted to connected spaces) and $\mathscr{B}$ imod $_{S}^{\text {gp }}$ are bicategorically equivalent with an induced equivalence on shadows. Under this equivalence (given in detail before [LM16, Thm. 5.1]) the map of parameterized spectra in (6.11) passes to the map of $\left(S, \Sigma_{+}^{\infty} \Omega X\right)$-bimodule spectra

$$
S \wedge S \Sigma_{+}^{\infty} \Omega X \rightarrow \Sigma_{+}^{\infty} \Omega X \wedge \Sigma_{+}^{\infty} \Omega X \Sigma_{+}^{\infty} \Omega X_{f} .
$$

The functoriality of the trace [Pon10] implies the following diagram commutes.

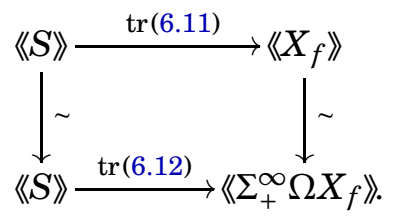

Then Proposition 6.9 implies

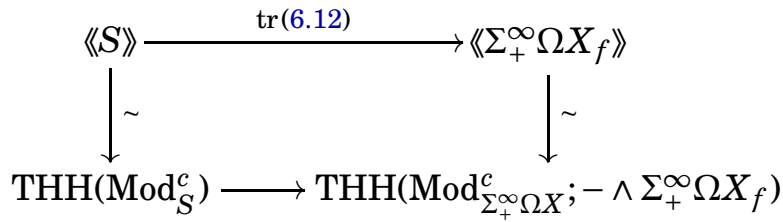

commutes and Corollary A.16 identifies

$$
\operatorname{THH}\left(\Sigma_{+}^{\infty} \Omega X ; \Sigma_{+}^{\infty} \Omega X_{f}\right) \simeq \Sigma_{+}^{\infty} \mathscr{L} X^{f} .
$$

\section{A $\pi_{0}$-LEVEL CYClOTOMIC TRACE}

Much of the motivation for the present paper comes from very concrete questions about THH. Above we used bicategories to understand the relationship between base change and traces using shadows, which yields information about THH. Here we consider a different, though related, question that will be essential for future work. Let $A$ be a ring spectrum, and let $f: P \rightarrow P$ be an endomorphism of an $A$-module $P$. Then $f$ determines an element of $\pi_{0}\left(\operatorname{THH}\left(\operatorname{Mod}_{A}^{c}\right)\right)$ as follows. First, it determines a map of modules $S \rightarrow \operatorname{End}(P)$ adjoint to $f$. This includes into the zero skeleton of $\mathrm{THH}\left(\operatorname{Mod}_{A}^{c}\right)$, which is

$$
\operatorname{sk}^{0}\left(\operatorname{THH}\left(\operatorname{Mod}_{A}^{c}\right)\right)=\bigvee_{M \in \operatorname{Mod}_{A}^{c}} \operatorname{End}(M)=\bigvee_{M \in \operatorname{Mod}_{A}^{c}} \operatorname{Mod}_{A}^{c}(M, M)
$$

The zero skeleton includes into THH( $\left.\operatorname{Mod}_{A}^{c}\right)$. Thus, we have a composite

$$
S \rightarrow \operatorname{End}_{\operatorname{Mod}_{A}^{c}}(P) \hookrightarrow \bigvee_{M \in \operatorname{Mod}_{A}^{c}} \operatorname{Mod}_{A}^{c}(M, M) \hookrightarrow \operatorname{THH}\left(\operatorname{Mod}_{A}^{c}\right)
$$

Finally, Morita invariance extends this map to

$$
S \rightarrow \operatorname{End}_{\operatorname{Mod}_{A}^{c}}(P) \hookrightarrow \bigvee_{M \in \operatorname{Mod}_{A}^{c}} \operatorname{Mod}_{A}^{c}(M, M) \hookrightarrow \operatorname{THH}\left(\operatorname{Mod}_{A}^{c}\right) \stackrel{\sim}{\rightarrow} \mathrm{THH}(A)
$$


yielding a map $S \rightarrow \mathrm{THH}(A)$, i.e. an element of $\pi_{0} \mathrm{THH}(A)$. The question is: What element of $\pi_{0} \mathrm{THH}(A)$ does $f$ determine? Intuitively, the answer should clearly be $\operatorname{tr}(f)$, but it is far from obvious given how we have defined it. It is therefore desirable to have an actual proof of this, and a categorical proof is even better. This is given in Proposition 7.4.

Remark 7.2. Since traces are additive, the association $[f] \mapsto \operatorname{tr}(f)$ given by the composite (7.1) descends to a map from the Grothendieck group of endomorphisms of $A$-modules, also called $K_{0}(\operatorname{End}(A))$. Thus, we obtain a map

$$
K_{0}(\operatorname{End}(A)) \rightarrow \pi_{0} \mathrm{THH}(A) .
$$

Indeed, connoiseurs will recognize (7.1) as the usual "inclusion of objects" map that is used to define the cyclotomic trace (see, e.g. [DM96]). We are only defining this at the level of $\pi_{0}$, so the usual difficulties don't intervene, but this is useful to keep in mind.

Since Proposition 7.4 is somewhat hard to parse, we motivate it by rephrasing the objects and maps involved in the trace in greater generality.

- The Morita equivalence

$$
\mathrm{THH}\left(\operatorname{Mod}_{A}^{c}\right) \stackrel{\sim}{\rightarrow} \mathrm{THH}(A)
$$

is implemented by a base change dual pair, so the last map in (7.1) is the Euler characteristic of some ( $\mathscr{C}, \mathscr{D})$-bimodule $\mathscr{M}$ for appropriate choices of $\mathscr{C}, \mathscr{D}, \mathscr{M}$.

- The middle term in (7.1), $\bigvee_{M \in \operatorname{Mod}_{A}^{c}} \operatorname{Mod}_{A}^{c}(M, M)$, is the shadow of a bimodule with trivial actions. That is, we've taken the shadow of the bimodule $\operatorname{Mod}_{A}^{c}$ where the usual left and right actions have been base changed to be trivial. Then the map

$$
\bigvee_{M \in \operatorname{Mod}_{A}^{c}} \operatorname{Mod}_{A}^{c}(M, M) \hookrightarrow \operatorname{THH}\left(\operatorname{Mod}_{A}^{c}\right)
$$

is the map induced on shadows from $\operatorname{Mod}_{A}^{c}$ with trivial left and right actions to $\operatorname{Mod}_{A}^{c}$ with the usual $\operatorname{Mod}_{A}^{c}$ actions.

If we rephrase this categorically, using the conventions above, $\operatorname{Mod}_{A}^{c}$ is $\mathscr{C}$ and the base change is implemented by a functor $F^{\prime}: \mathscr{A}^{\prime} \rightarrow \mathscr{C}$. The module with trivialized action is $F^{\prime} \mathscr{C}_{F^{\prime}}$ - note that this is a $\left(\mathscr{A}^{\prime}, \mathscr{A}^{\prime}\right)$-bimodule. Then the third map in (7.1) is the map

$$
\left\langle F_{F^{\prime}} \mathscr{C}_{F^{\prime}}\right\rangle \rightarrow\langle\langle\mathscr{C}\rangle
$$

induced by $F^{\prime}$. By Lemma 5.8 this map is $\left\langle\left\langle F^{\prime} \mathscr{C}_{F^{\prime}}\right\rangle \stackrel{\operatorname{tr}\left(\epsilon_{U_{\mathscr{C}}}^{F^{\prime}}\right)}{\longrightarrow}\langle\mathscr{C}\rangle\right\rangle$.

- In the inclusion

$$
\operatorname{End}_{\operatorname{Mod}_{A}^{c}}(P) \hookrightarrow \bigvee_{M} \operatorname{End}_{\operatorname{Mod}_{A}^{c}}(M)
$$

the module $\operatorname{End}_{\operatorname{Mod}_{A}^{c}}(P)$ is a shadow obtained by trivializing more of the action. Phrasing this categorically, $\operatorname{End}_{\operatorname{Mod}_{A}^{c}}(P)$ is $F_{F} \mathscr{C}_{F}$ where $F$ is a composite $\mathscr{A} \rightarrow \mathscr{A}^{\prime} \rightarrow \mathscr{C}$.

- Finally

$$
S \rightarrow \operatorname{End}_{\operatorname{Mod}_{A}^{c}}(P)
$$

is simply a map of spectra (i.e. $S$-modules). In the more categorical setting, this is a map of $(\mathscr{A}, \mathscr{A})$-bimodules $\mathscr{Q} \rightarrow{ }_{F} \mathscr{C}_{F}$.

To summarize, if we have an $(\mathscr{C}, \mathscr{D})$-bimodule $\mathscr{M}$, a functor $F: \mathscr{A} \rightarrow \mathscr{C}$ and a map of $(\mathscr{A}, \mathscr{A})$-bimodules $\mathscr{Q} \rightarrow{ }_{F} \mathscr{C}_{F}$, we have a map

$$
\left.\langle\mathscr{Q}\rangle \rightarrow\left\langle\left\langle F \mathscr{C}_{F}\right\rangle \stackrel{\operatorname{tr}\left(\epsilon_{U_{\mathscr{C}}}^{F}\right)}{\longrightarrow}\langle\mathscr{C}\rangle\right\rangle \stackrel{\chi(\mathscr{M})}{\longrightarrow}\langle\mathscr{D}\rangle\right\rangle .
$$


The first map comes from the 2-functoriality of shadows. Having placed ourself in a purely category theoretic context, we may do this nearly trivially. Many illuminating examples arise from specializations of the category theory including, of course, the main example.

Definition 7.3. If $\mathscr{M}$ is an $(\mathscr{C}, \mathscr{D})$-bimodule and $F: \mathscr{A} \rightarrow \mathscr{C}$ is a functor let $\epsilon_{\mathscr{C}}^{F, \mathscr{M}}$ be the $(\mathscr{A}, \mathscr{D})$-bimodule map given by composite

$$
{ }_{F} \mathscr{C}_{F} \odot_{F} \mathscr{M} \stackrel{\operatorname{id}_{F} \mathscr{C}_{F} \odot \sim}{ }{ }_{F} \mathscr{C}_{F} \odot{ }_{F} \mathscr{C} \odot \mathscr{M} \stackrel{\epsilon_{U_{\mathscr{C}}}^{F} \odot \mathrm{id} \mathscr{M}}{\longrightarrow}{ }_{F} \mathscr{C} \odot \mathscr{M} \stackrel{\sim}{\rightarrow} F \mathscr{M}
$$

This map is induced by the action of $\mathscr{C}$ on $\mathscr{M}$ restricted to the objects in the image of $F$.

Proposition 7.4. If $\mathscr{M}$ is a right dualizable $(\mathscr{C}, \mathscr{D})$-bimodule and $F: \mathscr{A} \rightarrow \mathscr{C}$ is an enriched functor, the trace of $\epsilon_{\mathscr{C}}^{F, \mathscr{M}}$ is the composite

$$
\left.\left\langle\mathscr{C}_{F}\right\rangle \stackrel{\operatorname{tr}\left(\epsilon_{U_{\mathscr{C}}}^{F}\right)}{\longrightarrow}\langle\mathscr{C}\rangle \stackrel{\chi(\mathscr{M})}{\longrightarrow}\langle\mathscr{D}\rangle\right\rangle
$$

If $\alpha: \mathscr{Q} \rightarrow{ }_{F} \mathscr{C}_{F}$ is a map of $(\mathscr{A}, \mathscr{A})$-bimodules the trace of $\mathscr{Q} \odot_{F} \mathscr{M} \stackrel{\alpha \odot \mathrm{id}_{F} \mathscr{M}}{\longrightarrow}{ }_{F} \mathscr{C}_{F} \odot$ $F \mathscr{M} \stackrel{\epsilon_{\mathscr{C}}^{F, \mathscr{M}}}{\longrightarrow} F \mathscr{M}$ is

$$
\left.\langle\mathscr{Q}\rangle \stackrel{\| \alpha\rangle}{\longrightarrow}\left\langle\mathscr{F}_{F} \mathscr{C}_{F}\right\rangle \stackrel{\operatorname{tr}\left(e_{U_{\mathscr{C}}}^{F}\right)}{\longrightarrow}\langle\mathscr{C}\rangle \stackrel{\chi(\mathscr{M})}{\longrightarrow}\langle\mathscr{D}\rangle\right\rangle .
$$

Proof. Using Theorem $3.18 \chi(\mathscr{M}) \circ \operatorname{tr}\left(\epsilon_{U_{\mathscr{C}}}^{F}\right)$ is the trace of the top row of the following commutative diagram.

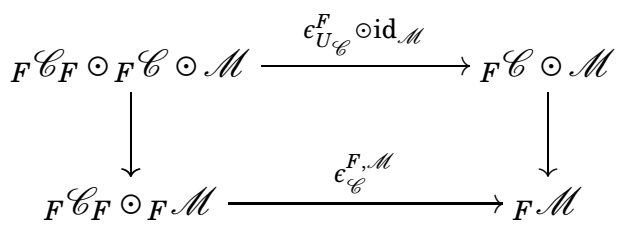

Since the vertical maps are identity maps on twisting objects and an isomorphism on the dualizable object the trace of $\epsilon_{U_{\mathscr{C}}}^{F} \odot \mathrm{id} \mathscr{M}_{\mathscr{C}}$ and $\epsilon_{\mathscr{C}}^{F, \mathscr{M}}$ are the same.

If $\mathscr{Q}$ is an $(\mathscr{A}, \mathscr{A})$-bimodule and $\alpha: \mathscr{Q} \rightarrow{ }_{F} \mathscr{C}_{F}$ is a module homomorphism, then Proposition 3.21 implies

$$
\langle\mathscr{Q}\rangle \stackrel{\langle\alpha\rangle}{\longrightarrow}\left\langle\left\langle\mathscr{C}_{F}\right\rangle \stackrel{\operatorname{tr}\left(\epsilon_{\mathscr{C}}^{F, \mathscr{M}}\right)}{\longrightarrow}\langle\mathscr{D}\rangle\right.
$$

is the trace of the composite $\mathscr{Q} \odot_{F} \mathscr{M} \stackrel{\alpha \odot \mathrm{id}_{F} \mathscr{M}}{\longrightarrow}{ }_{F} \mathscr{C}_{F} \odot_{F} \mathscr{M} \stackrel{\epsilon_{\mathscr{C}}^{F, \mathscr{M}}}{\longrightarrow} F \mathscr{M}$.

Before we consider examples of this result we need to fix some notation and give an example of Lemma 5.8. If $\mathscr{C}$ is a category enriched in $\mathscr{V}$, let $1_{\mathscr{C}}$ be the category whose objects are the objects of $\mathscr{C}$ and

$$
1_{\mathscr{C}}\left(c, c^{\prime}\right)=\left\{\begin{array}{cl}
1_{\mathscr{V}} & \text { if } c=c^{\prime} \\
\varnothing & \text { if } c \neq c^{\prime}
\end{array}\right.
$$

The composition map is the unit isomorphism. Let $I: 1_{\mathscr{C}} \rightarrow \mathscr{C}$ be the functor that picks out the identity map for each $c \in \mathscr{C}$. If $c$ is an object of $\mathscr{C}$, and we regard $1_{\mathscr{V}}$ as a one object category with object $*$, there is a functor $I_{c}: 1_{\mathscr{V}} \rightarrow 1_{\mathscr{C}} \rightarrow \mathscr{C}$ that picks out the identity map of $c$. As in Lemma 5.8, the following diagrams commute.

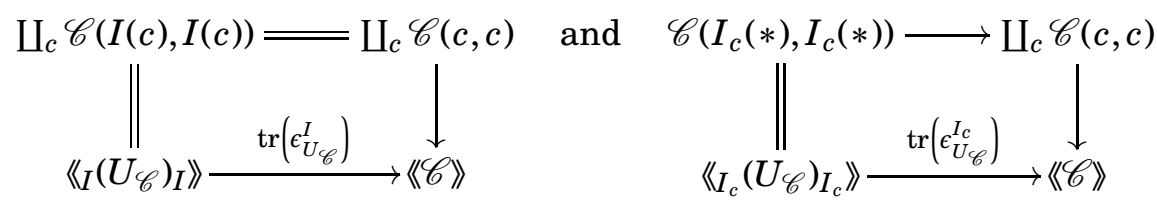


These commuting squares provide the necessary connection between maps of endomorphisms and traces.

Example 7.6. If $\mathscr{V}$ is the category of abelian groups and categories $\mathscr{C}$ and $\mathscr{D}$ each have a single object, $\mathscr{C}$ is a ring $C, \mathscr{D}$ is a ring $D$. An $(\mathscr{C}, \mathscr{D})$-bimodule $\mathscr{M}$ is an $(C, D)$-bimodule $M$. If $i: \mathbb{Z} \rightarrow C$ is the map that picks out the monoidal unit,

$$
\epsilon_{C}^{i}:{ }_{i} C_{i} \otimes{ }_{i} C \rightarrow{ }_{i} C
$$

is the ring multiplication in $C$ and

$$
\epsilon_{C}^{i, M}:{ }_{i} C_{i} \otimes{ }_{i} M \rightarrow{ }_{i} M
$$

is the module structure map. If $f: A \rightarrow C$ is a ring homomorphism, and $M$ is an $(C, D)$ bimodule

$$
\epsilon_{A}^{i,{ }_{f} M}:{ }_{i} A_{i} \otimes{ }_{i}\left({ }_{f} M\right) \rightarrow{ }_{i}\left({ }_{f} M\right)
$$

is given by $\epsilon_{A}^{i, f} M(a, m)=f(a) m$.

Proposition 7.4 implies the trace of the module structure map $\epsilon_{C}^{i, M}:{ }_{i} C_{i} \otimes{ }_{i} M \rightarrow{ }_{i} M$ is the composite

$$
\left.\left\langle i C_{i}\right\rangle \rightarrow\langle C\rangle\right\rangle \stackrel{\chi(M)}{\longrightarrow}\langle\langle D\rangle
$$

The commuting diagram in (7.5) identifies the first map as the map $C \rightarrow\langle C\rangle$.

If $\alpha: \mathbb{Z} \rightarrow{ }_{i} C_{i}$ is a module homomorphism, the composite

$$
M \cong \mathbb{Z} \otimes_{i} M \stackrel{\alpha \otimes \mathrm{id}}{\longrightarrow}{ }_{i} C_{i} \otimes_{i} M \stackrel{\epsilon_{C}^{i, M}}{\longrightarrow}{ }_{i} M
$$

is given by $n \mapsto \alpha(1) n$. While this is very similar to the description of $\epsilon_{A}^{i_{f} M}$, since $\alpha$ is a module homomorphism there is greater flexibility in the image of 1 .

Proposition 7.4 implies the trace of

$$
\mathbb{Z} \odot{ }_{i} M \stackrel{\alpha \odot \mathrm{id}_{i} M}{\longrightarrow}{ }_{i} C_{i} \odot{ }_{i} M \stackrel{\epsilon_{C}^{i, M}}{\longrightarrow}{ }_{i} M
$$

is $\left.\langle\mathbb{Z}\rangle \stackrel{\| \alpha\rangle\rangle}{\longrightarrow}\left\langle{ }_{i} C_{i}\right\rangle \stackrel{\operatorname{tr}\left(\epsilon_{C}^{i}\right)}{\longrightarrow}\langle C\rangle\right\rangle \stackrel{\chi(M)}{\longrightarrow}\langle\langle D\rangle$.

While we focused on the case where $\mathscr{V}$ is the category of abelian groups, Example 7.6 holds as long as objects of $\mathscr{V}$ have underlying sets.

Corollary 7.7. For a functor $F: \mathscr{A} \rightarrow \mathscr{C}$, a right dualizable $(\mathscr{C}, \mathscr{D})$-bimodule $\mathscr{M}$, an object $a \in \mathscr{A}$ and a map of $\left(1_{\mathscr{V}}, 1_{\mathscr{V}}\right)$-bimodules $\alpha: 1_{\mathscr{V}} \rightarrow I_{a} \mathscr{A}_{I_{a}}$ the trace of

$$
1_{\mathscr{V}} \odot_{F \circ I_{a}} \mathscr{M} \stackrel{\alpha \odot \operatorname{id}_{F_{\circ} I_{a}} \mathscr{M}}{\longrightarrow} I_{a} \mathscr{A} I_{a} \odot \odot_{F \circ I_{a}} \mathscr{M} \cong I_{I_{a}} \mathscr{A}_{I_{a}} \odot_{I_{a}}(F \mathscr{M}) \stackrel{\epsilon_{U_{\mathscr{A}}}^{I_{a}, F}}{\longrightarrow} I_{a}(F \mathscr{M}) \cong{ }_{F \circ I_{a}} \mathscr{M}
$$

as a map of $\left(1_{\mathscr{V}}, \mathscr{D}\right)$-bimodules is

$$
\left.\left.1_{\mathscr{V}} \stackrel{\alpha}{\rightarrow}\left\langle I_{a} \mathscr{A} I_{a}\right\rangle \stackrel{\operatorname{tr}\left(\epsilon_{U_{\mathscr{D}}}^{I_{a}}\right)}{\longrightarrow}\langle\mathscr{A}\rangle\right\rangle \stackrel{\chi(F \mathscr{M})}{\longrightarrow}\langle\mathscr{D}\rangle\right\rangle .
$$

Proof. This follows from Proposition 7.4 with the substitutions

\begin{tabular}{c|c|c|c|c|c}
$\mathscr{A}$ & $\mathscr{C}$ & $\mathscr{D}$ & $\mathscr{M}$ & $F$ & $\mathscr{Q}$ \\
\hline $1_{\mathscr{V}}$ & $\mathscr{A}$ & $\mathscr{D}$ & $F \mathscr{M}$ & $I_{a}$ & $1_{\mathscr{V}}$
\end{tabular}

Theorem 7.8. For a Morita equivalence $E_{a}: \operatorname{End}_{\mathscr{A}}(a) \rightarrow \mathscr{A}$, an object $b$ of $\mathscr{A}$, and a map of modules $\alpha: 1_{\mathscr{V}} \rightarrow I_{b} \mathscr{A}_{I_{b}}$, the trace of

$$
1_{\mathscr{V}} \odot I_{b} \mathscr{A}_{E_{a}} \stackrel{\alpha \odot \mathrm{id}}{\longrightarrow} I_{b} \mathscr{A}_{I_{b}} \odot I_{b} \mathscr{A}_{E_{a}} \stackrel{\epsilon_{U_{\mathscr{A}}}^{I_{b}, \mathscr{A}} E_{a}}{\longrightarrow} I_{b} \mathscr{A} E_{a}
$$


is the composite

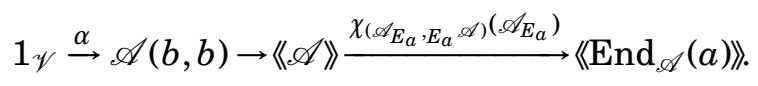

Proof. This follows from Proposition 7.4 with the substitutions

\begin{tabular}{c|c|c|c|c|c}
$\mathscr{A}$ & $\mathscr{C}$ & $\mathscr{D}$ & $\mathscr{M}$ & $F$ & $\mathscr{Q}$ \\
\hline $\operatorname{End}_{\mathscr{A}}(b)$ & $\mathscr{A}$ & $\operatorname{End}_{\mathscr{A}}(a)$ & $\mathscr{A}_{E_{a}}$ & $I_{b}$ & $1_{\mathscr{V}}$
\end{tabular}

Example 7.9. Let $\mathscr{V}$ be the category of abelian groups. For a ring $C$ and a finitely generated projective $C$-module $P$, an endomorphism $f: P \rightarrow P$ is represented by a map of abelian groups $\mathbb{Z} \stackrel{[f]}{\longrightarrow} \operatorname{Hom}(P, P)$, i.e. a map of $\mathbb{Z}$-modules. Despite the fact that $\operatorname{Hom}(P, P)$ has the structure of a monoid, the map is not a map of monoids.

Applying Theorem 7.8 with the substitutions

the composite

$$
\begin{array}{c|c|c|c}
\mathscr{A} & a & b & \alpha \\
\hline \operatorname{Mod}_{C}^{c} & C & P & {[f]}
\end{array}
$$

$$
\mathbb{Z} \stackrel{[f]}{\longrightarrow} \operatorname{Mod}_{C}^{c}(P, P) \rightarrow\left\langle\left\langle\operatorname{Mod}_{C}^{c}\right\rangle \stackrel{\chi_{\left(\operatorname{Mod}_{C}^{c}\right) E_{C}, E_{C}\left(\operatorname{Mod}_{C}^{c}\right)}\left(\left(\operatorname{Mod}_{C}^{c}\right)_{E_{C}}\right)}{\longrightarrow}\left\langle\operatorname{End}_{\operatorname{Mod}_{C}^{c}}(C)\right\rangle\right.
$$

is the trace of

$$
\operatorname{Mod}_{C}^{c}(P, C) \cong \mathbb{Z} \otimes \operatorname{Mod}_{C}^{c}(P, C) \stackrel{[f] \odot \mathrm{id}}{\longrightarrow} \operatorname{End}_{\operatorname{Mod}_{C}^{c}}(P) \otimes \operatorname{Mod}_{C}^{c}(P, C) \stackrel{\epsilon_{U_{\operatorname{Mod}_{C}^{c}}}^{\epsilon_{P},\left(\operatorname{Mod}_{C}^{c}\right)_{E}}}{\longrightarrow} \operatorname{Mod}_{C}^{c}(P, C) .
$$

Following Example 7.6 the second map is the action of $\operatorname{End}_{M_{C} d_{C}^{c}}(P)$ on $\operatorname{Mod}_{C}^{c}(P, C)$ by composition, and so the the image of $\psi \in \operatorname{Mod}_{C}^{c}(P, C)$ in $\operatorname{Mod}_{C}^{c}(P, C)$ is $\psi \circ f$. So (7.10) is the dual of $f$ and the trace of (7.10) is the trace of $f$ as in Remark 3.12.

With Theorem 7.8 in place, we can state one of the main theorems of this paper, concerning the relationship between the cyclotomic trace and the bicategorical trace.

Theorem 7.11. Let $R$ be a ring spectrum, and $f: M \rightarrow M$ an endomorphism of compact $R$-modules. Then the composition

$$
S \stackrel{f}{\rightarrow} \operatorname{Mod}_{R}^{c}(M, M) \hookrightarrow \bigvee_{M} \operatorname{Mod}_{R}^{c}(M, M) \hookrightarrow \operatorname{THH}\left(\operatorname{Mod}_{R}^{c}\right) \stackrel{\chi}{\rightarrow} \mathrm{THH}(R)
$$

coincides with the bicategorical trace $S \rightarrow \mathrm{THH}(R)$ induced by $f: M \rightarrow M$.

Proof. We use Theorem 7.8 with the substitutions

$$
\begin{array}{c|c|c|c|c}
\mathscr{A} & a & b & 1_{\mathscr{V}} & \alpha \\
\hline \operatorname{Mod}_{R}^{c} & R & M & S & f .
\end{array}
$$

\section{ApPENDiX A. IDENTIFYING $\mathscr{L} X^{f}$ VIA THH}

This appendix is devoted to performing a computation that is necessary for Theorem 6.10. We note that this computation is not strictly necessary since Theorem A.14 can be deduced from structural results. However, the inclusion of this exposition is useful for understanding geometrically $w h y$ the twisted free loop space should be appearing.

Given a map $f: X \rightarrow X$ we identify the twisted THH spectrum $\operatorname{THH}\left(\Sigma_{+}^{\infty} \Omega X ; \Sigma_{+}^{\infty} \Omega X_{f}\right)$ with a twisted free loop space $\mathscr{L} X^{f}$. It is probable that computations of this sort for 
arbitrary self-maps of ring spectra, $g: A \rightarrow A$, are interesting; but we content ourselves with the current example.

It is a classical fact due to Goodwillie [Goo85] that the cyclic bar construction applied to the based loop space is the free loop space: $B^{\text {cy }}(\Omega X) \simeq \mathscr{L} X$. In modern categories of spectra, the suspension spectrum functor interacts nicely with bar constructions [EKMM97], and so this provides a computation of the topological Hochschild homology of the "spectral group ring" $\Sigma_{+}^{\infty} \Omega X$ :

$$
\operatorname{THH}\left(\Sigma_{+}^{\infty} \Omega X\right) \simeq \Sigma_{+}^{\infty} \mathscr{L} X
$$

In the bar construction above, $\Omega X$ is considered as an $(\Omega X, \Omega X)$-bimodule in the obvious way. In what follows, we consider $\Omega X$ as an $(\Omega X, \Omega X)$-bimodule with the action twisted by an endomorphism $f: X \rightarrow X$. That is, let $\omega \in \Omega X$ and let $\gamma$ be the path from $*$ to $f(*)$, then we define the left action of $\omega^{\prime} \in \Omega X$ by $\omega^{\prime} * \omega$ and the right action by $\omega * \gamma * f\left(\omega^{\prime}\right) * \gamma^{-1}$. Let $\Omega X^{f}$ be $\Omega X$ with this bimodule structure; we may then ask about $B^{\text {cy }}\left(\Omega X ; \Omega X^{f}\right)$. We compute this below and show it to be homotopy equivalent to a twisted version of the free loop space, which is the proper receptacle for the Reidemeister trace.

The computation proceeds mostly as in [Goo85]. We compare the cyclic bar construction as a simplicial space with the singular simplicial space of the twisted free loop space. In order to work with strict topological monoids and bimodules we need to work with Moore path spaces, Moore loop spaces, etc. Also, the introduction of $\gamma$ above in order to transport between loops based at $*$ and loops based at $f(*)$ is unfortunate and we avoid it below by choosing two different models for $\Omega X$ when it suits us.

Definition A.1. The free Moore path space is defined to be

$$
\mathscr{P}^{M} X=\{(\gamma, u) \in \operatorname{Map}([0, \infty), X) \times[0, \infty): \gamma(t)=\gamma(u) t \geq u\} .
$$

Here $\operatorname{Map}([0, \infty), X)$ is given the compact open topology. The space $\mathscr{P}^{M} X$ comes equipped with two maps $s: \mathscr{P}^{M} X \rightarrow X$ and $t: \mathscr{P}^{M} X \rightarrow X$ given by evaluation at 0 and $u$.

Definition A.2. Let $X$ be a based space with base $*$. Then the Moore loop space is

$$
\Omega^{M} X=\left\{(\gamma, u) \in \mathscr{P}^{M} X: \gamma(0)=\gamma(u)=*\right\}
$$

that is, it is the pullback

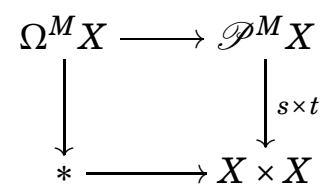

For work below, it is convenient to denote the "length" of the path, $u$, by $|\omega|$.

For use in the two-sided bar construction, we need another version of this loop space. The following construction allows us to act on the left by loops based at $*$ and on the right by loops based at $f(*)$.

Definition A.3. The $f$-twisted Moore loop space is the topological space

$$
\Omega^{M} X^{f}=\left\{(\gamma, u) \in \mathscr{P}^{M} X: \gamma(0)=*, \gamma(u)=f(*)\right\}
$$

i.e. it is given as a pullback

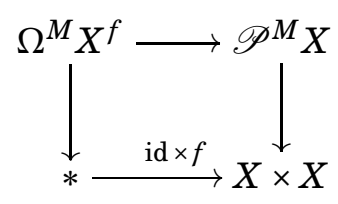

Again, $|\omega|$ denotes the length of the path $\omega$. 
This space $\Omega^{M} X^{f}$ is homotopy equivalent to $\Omega^{M} X$ and has the structure of an $\left(\Omega^{M} X, \Omega^{M} X\right)$-bimodule. For $\alpha \in \Omega^{M} X, \gamma \in \Omega^{M} X^{f}$ and $\beta \in \Omega^{M} X$

$$
\alpha \cdot \gamma=\alpha * \gamma \quad \gamma \cdot \beta=\gamma * f(\beta)
$$

For our definition of the free loop space, it is more convenient to use the naive definition, not the Moore-style definition. We let $\mathscr{P} X$ be paths in $X$ and $\Omega X$ be loops in $X$.

Definition A.4. The $f$-twisted free loop space is defined to be

$$
\mathscr{L} X^{f}=\{\gamma \in \mathscr{P} X: \gamma(0)=f(\gamma(1))\}
$$

That is, $\mathscr{L} X^{f}$ arises as the pullback

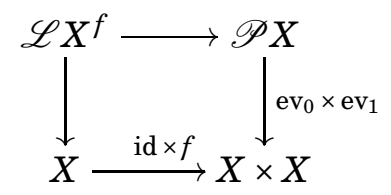

Consequently, $\mathscr{L}^{f} X$ sits in a fibration sequence

$$
\Omega X^{f} \rightarrow \mathscr{L}^{f} X \rightarrow X
$$

We can now define the main object of concern in this section.

Definition A.5. $N^{\text {cy }}\left(\Omega^{M} X, \Omega^{M} X^{f}\right)$ is the geometric realization of the simplicial space with $n$-simplices

$$
N_{n}^{\text {cy }}\left(\Omega^{M} X, \Omega^{M} X^{f}\right)=\underbrace{\Omega^{M} X \times \cdots \times \Omega^{M} X}_{n \text { times }} \times \Omega^{M} X^{f}
$$

The face maps $d_{i}: N_{n}^{\mathrm{cy}} \rightarrow N_{n-1}^{\mathrm{cy}}$ are given by

$$
d_{i}\left(\omega_{0}, \ldots, \omega_{n-1}, \omega\right)= \begin{cases}\left(\omega_{0}, \ldots, \omega_{i} \omega_{i+1}, \ldots, \omega_{n-1}, \omega\right) & i<n \\ \left(\omega_{0}, \ldots, \omega_{n-1} \omega\right) & i=n \\ \left(\omega_{1}, \ldots, \omega_{n-1}, \omega f\left(\omega_{0}\right)\right) & i=n+1\end{cases}
$$

The degeneracies $s_{j}: N_{n}^{c y} \rightarrow N_{n+1}^{\mathrm{cy}}$ are given by the insertion of trivial paths:

$$
s_{j}\left(\omega_{0}, \ldots, \omega_{n-1}, \omega\right)=\left(\cdots, \omega_{j}, *, \omega_{j+1}, \ldots\right)
$$

Remark A.6. Level-wise this is equivalent to the cyclic bar construction that computes $\mathscr{L} X$, but the face maps are different.

In order to proceed with the comparison, we need to define various simplicial spaces. In what follows elements in $\Delta^{n}$ referred to by the barycentric coordinates $\left(u_{0}, \ldots, u_{n}\right)$.

The $\Delta^{n}$ are assembled into a cosimplicial space $\Delta^{\bullet}$ via the cosimplicial maps

$$
\begin{aligned}
d_{i}\left(u_{0}, \ldots, u_{n}\right) & =\left(u_{0}, \ldots, u_{i}+u_{i+1}, \ldots, u_{n}\right) \\
s_{i}\left(u_{0}, \ldots, u_{n}\right) & =\left(u_{0}, \ldots, u_{i}, 0, u_{i+1}, \ldots, u_{n}\right)
\end{aligned}
$$

For $\gamma \in \operatorname{Map}\left(\Delta^{n}, \mathscr{P} X\right)$ and $\left(u_{0}, \ldots, u_{n}\right) \in \Delta^{n}, \gamma_{u_{0}, \ldots, u_{n}}$ denotes the result of evaluating $\gamma$ at $\left(u_{0}, \ldots, u_{n}\right)$.

Definition A.7. The space $\left(\Omega X^{f}\right)^{\Delta^{n}}$ is defined to be

$$
\left(\Omega X^{f}\right)^{\Delta^{n}}=\left\{\gamma \in \operatorname{Map}\left(\Delta^{n}, \mathscr{P} X\right) \mid \begin{array}{c}
\gamma_{u_{0}, \ldots, u_{n}}(0)=*, \gamma_{u_{0}, \ldots, u_{n}}(1)=f(*) \\
\text { for all }\left(u_{0}, \ldots, u_{n}\right) \in \Delta^{n}
\end{array}\right\}
$$

The cosimplicial structure on $\Delta^{\bullet}$ induces a simplicial structure on $\left(\Omega X^{f}\right)^{\Delta^{\bullet}}$. The face maps are given by restriction:

$$
d_{i}(\gamma)\left(t, u_{0}, \ldots, u_{n}\right)=\gamma\left(t, u_{0}, \ldots, u_{i}, 0, u_{i+1}, \ldots, u_{n}\right)
$$


where $t$ is the path coordinate and the degeneracy maps are given by addition of coordinates:

$$
s_{i}(\gamma)\left(t, u_{0}, \ldots, u_{n}\right)=\gamma\left(t, u_{0}, \ldots, u_{i}+u_{i+1}, \ldots, u_{n}\right)
$$

Definition A.8. The topological space $\left(\mathscr{L} X^{f}\right)^{\Delta^{n}}$ is defined to be

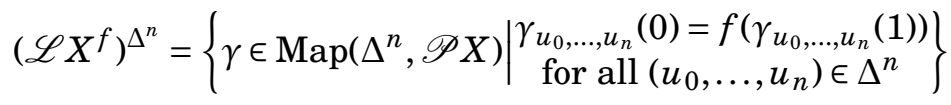

Again, the cosimplicial structure on $\Delta^{\bullet}$ induces a simplicial structure on $\left(\mathscr{L} X^{f}\right)^{\Delta^{\bullet}}$. The face and degeneracy maps are as above.

The idea is now to compare the bar constructions with known fibration sequences. Consider the following diagram.

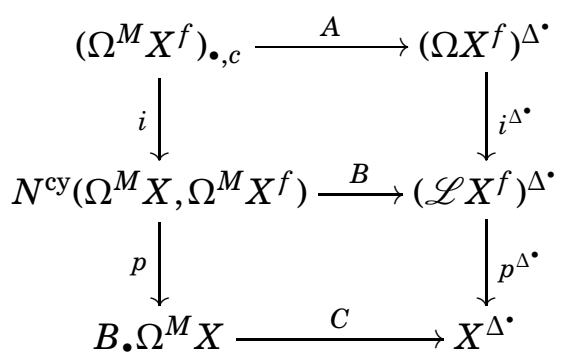

The upper left corner is the constant simplicial space $\left(\Omega^{M} X^{f}\right)_{\bullet, c}$, and the lower left corner is the usual bar construction on the topological monoid $\Omega^{M} X$. The right side of the diagram is simply the singular simplicial spaces of the fibration $\Omega X^{f} \rightarrow \mathscr{L} X^{f} \rightarrow X$.

The left column will be a fibration sequence upon geometric realization, the right hand side is trivially so, and we show that all horizontal maps are weak equivalences following [Goo85].

We now write out all of the maps in the diagram:

- The map

$$
A:\left(\Omega^{M} X^{f}\right)_{n, c} \rightarrow\left(\Omega X^{f}\right)^{\Delta^{n}}
$$

is given by reparamterizing the loops:

$$
A(\omega)\left(t, u_{0}, \ldots, u_{n}\right)=\omega(t|\omega|)
$$

- The map

$$
B: N_{n}^{\mathrm{cy}}\left(\Omega^{M} X, \Omega^{M} X^{f}\right) \rightarrow\left(\mathscr{L} X^{f}\right)^{\Delta^{n}}
$$

is given by:

$$
\begin{aligned}
& B\left(\omega_{0}, \omega_{1}, \ldots, \omega_{n-1}, \omega\right)\left(t, u_{0}, \ldots, u_{n}\right) \\
& =\widetilde{\omega}\left(t\left(\left|\omega_{0}\right|+\left|\omega_{1}\right|+\cdots+\left|\omega_{n-1}\right|+|\omega|\right)\right. \\
& \left.+u_{0}\left(\left|\omega_{0}\right|+\cdots+\left|\omega_{n-1}\right|\right)+u_{1}\left(\left|\omega_{1}\right|+\cdots+\left|\omega_{n-1}\right|\right) \cdots+u_{n-1}\left(\left|\omega_{n-1}\right|\right)\right)
\end{aligned}
$$

where

$$
\widetilde{\omega}=\omega_{0} * \cdots * \omega_{n-1} * \omega * f\left(\omega_{0}\right) * f\left(\omega_{1}\right) \cdots \cdots * f\left(\omega_{n-1}\right)
$$

- The map

$$
C: B_{n} \Omega^{M} X \rightarrow X^{\Delta^{n}}
$$



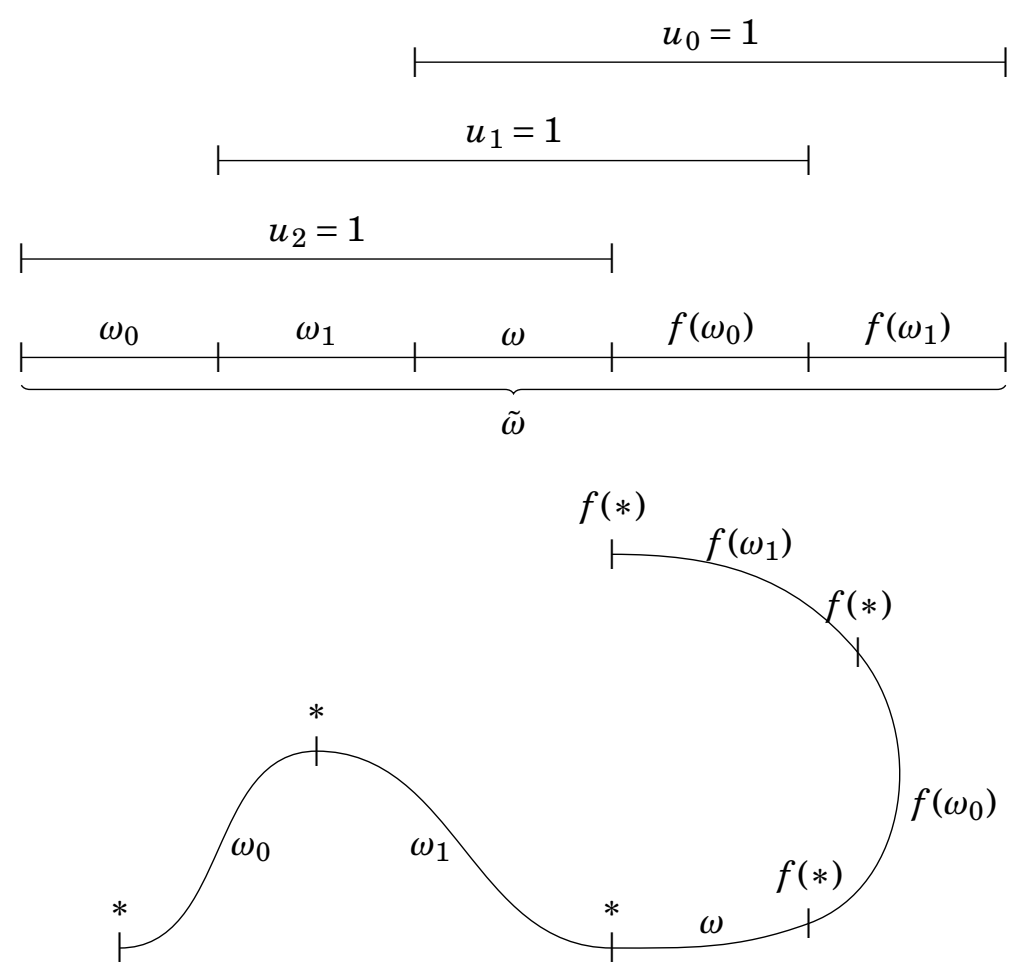

Figure A.10. The map $B$

is given by

$$
\begin{aligned}
& C\left(\omega_{0}, \ldots, \omega_{n-1}\right)\left(u_{0}, \ldots, u_{n}\right) \\
& =\left(\omega_{1} * \cdots * \omega_{n}\right)\left(u_{0}\left(\left|\omega_{0}\right|+\cdots+\left|\omega_{n-1}\right|\right)+u_{1}\left(\left|\omega_{1}\right|+\cdots+\left|\omega_{n-1}\right|\right)+\cdots+u_{n}\left(\left|\omega_{n-1}\right|\right)\right)
\end{aligned}
$$

- The maps $i^{\Delta^{\bullet}}$ and $p^{\Delta^{\bullet}}$ the obvious ones induced by maps on spaces.

- The map $i$. is given level-wise as

$$
i_{n}(\omega)=(*, \ldots, *, \omega)
$$

and $p$. is given level-wise as

$$
p_{n}\left(\omega_{0}, \ldots, \omega_{n-1}, \omega_{n}\right)=\left(\omega_{0}, \ldots, \omega_{n-1}\right)
$$

The definition of the map, $B$, above is somewhat elaborate. The example in Figure A.10 hopefully elucidates it.

Example A.11. We consider the case when $n=2$ for the map $B$ above, which is enough to capture the issues. We define

$$
\widetilde{\omega}=\omega_{0} * \omega_{1} * \omega * f\left(\omega_{0}\right) * f\left(\omega_{1}\right)
$$

and a family of paths parameterized by the 2 -simplex

$$
\Delta^{2}=\left\{\left(u_{0}, u_{1}, u_{2}\right): u_{0}+u_{1}+u_{2}=1\right\} .
$$

We think of this simplex as interpolating between three extremes: the cases when each of $u_{0}, u_{1}$ and $u_{2}$ are 1 . We'd like the extreme cases to be

- $u_{2}=1$ - the path $\omega_{0} * \omega_{1} * \omega$

- $u_{1}=1$ - the path $\omega_{1} * \omega * f\left(\omega_{0}\right)$

- $u_{0}=1$ - the path $\omega * f\left(\omega_{0}\right) * f\left(\omega_{1}\right)$. 
We would also like that if the path starts at $x$, the endpoint is $f(x)$. Consider the path

$$
\widetilde{\omega}\left(t\left(\left|\omega_{0}\right|+\left|\omega_{1}\right|+|\omega|\right)+u_{0}\left(\left|\omega_{0}\right|+\left|\omega_{1}\right|\right)+u_{1}\left(\left|\omega_{0}\right|\right)\right)
$$

We can check that when $u_{2}=1$ then $u_{0}, u_{1}=0$ and the above gives $\widetilde{\omega}\left(t\left(\left|\omega_{0}\right|+\left|\omega_{1}\right|+|\omega|\right)\right)$ which is exactly $\omega_{0} * \omega_{1} * \omega$. When $u_{1}=1$, then the above is $\widetilde{\omega}\left(t\left(\left|\omega_{0}\right|+\left|\omega_{1}\right|\right)+\left|\omega_{0}\right|\right)$, which is the path $\omega_{1} * \omega * f\left(\omega_{0}\right)$. Finally, when $u_{0}=1$ we get the path $\omega * f\left(\omega_{0}\right) * f\left(\omega_{1}\right)$.

We also note that for any choice of $u_{0}, u_{1}$,

$$
\begin{aligned}
& \widetilde{\omega}\left(u_{0}\left(\left|\omega_{0}\right|+\left|\omega_{1}\right|\right)+u_{1}\left(\left|\omega_{0}\right|\right)\right. \\
& =\widetilde{\omega}\left(\left|\omega_{0}\right|+\left|\omega_{1}\right|+|\omega|+u_{0}\left(\left|\omega_{0}\right|+\left|\omega_{1}\right|\right)+u_{1}\left(\left|\omega_{0}\right|\right)\right)
\end{aligned}
$$

essentially by definition, so that each path parameterized by $u_{0}, u_{1}, u_{2}$ is in $\mathscr{L} X^{f}$.

There are a few things we need to check about the maps above. First, we should check that $B$ is actually a map to $\left(\mathscr{L} X^{f}\right)^{\Delta^{*}}-$ that is, check that the endpoints are correct. The argument is the same as in the example.

Lemma A.12. The map $B$ defined above, is well-defined.

Second, it is easy to observe that the diagram actually commutes. Third, it is clear that $A$ and $C$ are simplicial maps. Though it is an irritating exercise, it is easy to check that $B$ is as well.

Proposition A.13. $B: N^{c y}\left(\Omega^{M} X, \Omega^{M} X^{f}\right) \rightarrow\left(\mathscr{L} X^{f}\right)^{\Delta^{\bullet}}$ is a simplicial map.

The rest of the proof proceeds as in [Goo85, Sect. V]. Upon geometric realization, the left hand column of A.9 becomes a fibration up to homotopy by [Seg74], as does the right hand column. To prove that $B$ is a weak equivalence, it thus suffice to prove that $A, C$ are weak equivalences. This is done in [Goo85, Sect. V].

All of this work entitles us to the following theorem.

Theorem A.14. Let $X$ be a (connected) topological space. Then

$$
B^{c y}\left(\Omega^{M} X, \Omega^{M} X^{f}\right) \simeq \mathscr{L} X^{f}
$$

As a corollary when $f=\operatorname{Id}$ we recover Goodwillie's original computation.

Corollary A.15. [Goo85, Sect. V.1] For a topological space, the geometric realization of the cyclic bar construction on the based loop space is equivalent to the free loop space:

$$
B^{c y}(\Omega X) \simeq \mathscr{L} X
$$

and for $X=B G$ where $G$ is a finite group

$$
B^{c y}(G) \simeq \mathscr{L} B G
$$

As another corollary, we have a generalization of the classical computation

$$
\operatorname{THH}\left(\Sigma_{+}^{\infty} \Omega X\right) \simeq \Sigma_{+}^{\infty} \mathscr{L} X .
$$

\section{Corollary A.16.}

$$
\operatorname{THH}\left(\Sigma_{+}^{\infty} \Omega X ; \Sigma_{+}^{\infty} \Omega X^{f}\right) \simeq \Sigma_{+}^{\infty} \mathscr{L} X^{f}
$$

The space $\mathscr{L} X^{f}$ is the space of homotopy fixed points of a self-map $f: X \rightarrow X$, computed by taking a "derived intersection" of $X$ and the image of $X$ under $f$. One could wish to have a similar THH description of the derived intersection of two maps $f, g: X \rightarrow Y$. The following corollary is a more general statement, and easy corollary of the proof of Theorem A.14. Though we do not use this generality in the paper, it is useful to record for later work. 
Corollary A.17. Let $f, g: X \rightarrow Y$ be self maps and let $\mathscr{L} Y^{f, g}$ be the homotopy pullback

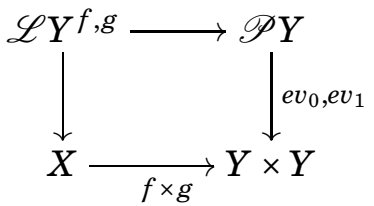

Similarly, let $\Sigma_{+}^{\infty} \Omega Y^{f, g}$ be the $\left(\Sigma_{+}^{\infty} \Omega Y, \Sigma_{+}^{\infty} \Omega Y\right)$-bimodule $\Sigma_{+}^{\infty} \Omega X$ with left action by $f$ and right action by $g$. Then

$$
\operatorname{THH}\left(\Sigma_{+}^{\infty} \Omega X ; \Sigma_{+}^{\infty} \Omega X^{f, g}\right) \simeq \Sigma_{+}^{\infty} \mathscr{L} Y^{f, g} .
$$

Proof. The proof is identical to that of Theorem A.14

\section{REFERENCES}

[AS68] M. F. Atiyah and I. M. Singer, The index of elliptic operators. I, Ann. of Math. (2) 87 (1968), 484-530. doi:0.2307/1970715 (cit. on p. 1).

[Bar16] C. Barwick, On the algebraic K-theory of higher categories, J. Topol. 9 (2016), no. 1, $245-347$. doi:10.1112/jtopol/jtv042 arXiv:1204.3607 (cit. on p. 2).

[Bén67] J. Bénabou, Introduction to bicategories, Reports of the Midwest Category Seminar, Springer, Berlin, 1967, pp. 1-77. (cit. on p. 4).

[BM90] S. Bentzen and I. Madsen, Trace maps in algebraic K-theory and the Coates-Wiles homomorphism, J. Reine Angew. Math. 411 (1990), 171-195. doi:10.1515/crll.1990.411.171 (cit. on p. 23).

[BGT13] A. J. Blumberg, D. Gepner, and G. Tabuada, A universal characterization of higher algebraic K-theory, Geom. Topol. 17 (2013), no. 2, 733-838. doi:10.2140/gt.2013.17.733 arXiv:1001.2282 (cit. on p. 2).

[BM12] A. J. Blumberg and M. A. Mandell, Localization theorems in topological Hochschild homology and topological cyclic homology, Geom. Topol. 16 (2012), no. 2, 1053-1120. doi:10.2140/gt.2012.16.1053 arXiv:0802.3938 (cit on pp. 5, 6, 8, 19).

[BHM93] M. Bökstedt, W. C. Hsiang, and I. Madsen, The cyclotomic trace and algebraic K-theory of spaces, Invent. Math. 111 (1993), no. 3, 465-539. doi:10.1007/BF01231296 (cit. on p. 2).

[Bök] M. Bökstedt, Topological Hochschild Homology, Preprint. (cit. on p. 5).

[Dol65] A. Dold, Fixed point index and fixed point theorem for Euclidean neighborhood retracts, Topology 4 (1965), 1-8. doi:10.1016/0040-9383(65)90044-3 (cit. on p. 10).

[DP80] A. Dold and D. Puppe, Duality, trace, and transfer, Proceedings of the International Conference on Geometric Topology (Warsaw, 1978) (Warsaw), PWN, 1980, pp. 81-102. (cit. on p. 10).

[DM96] B. I. Dundas and R. McCarthy, Topological Hochschild homology of ring functors and exact categories, J. Pure Appl. Algebra 109 (1996), no. 3, 231-294. doi:10.1016/0022-4049(95)00089-5 (cit on pp. 5, 19, 27).

[EKMM97] A. D. Elmendorf, I. Kriz, M. A. Mandell, and J. P. May, Rings, modules, and algebras in stable homotopy theory, Mathematical Surveys and Monographs, vol. 47, American Mathematical Society, Providence, RI, 1997, With an appendix by M. Cole. (cit. on p. 31).

[Gh66] S. Gen-hua, On least number of fixed points and Nielsen numbers, Chinese Math.-Acta 8 (1966), 234-243. (cit. on p. 2).

[Goo85] T. G. Goodwillie, Cyclic homology, derivations, and the free loopspace, Topology 24 (1985), no. 2, 187-215. doi:10.1016/0040-9383(85)90055-2 (cit on pp. 31, 33, 35).

[Hat65] A. Hattori, Rank element of a projective module, Nagoya Math. J. 25 (1965), 113-120. (cit. on p. 10).

[Iwa99] Y. Iwashita, The Lefschetz-Reidemesiter trace in algebraic k-theory, Ph.D. thesis, University of Illinois at Urbana-Champaign, 1999. (cit. on p. 4).

[Kal15] D. Kaledin, Trace theories and localization, Stacks and categories in geometry, topology, and algebra, Contemp. Math., vol. 643, Amer. Math. Soc., Providence, RI, 2015, pp. 227-262. doi:10.1090/conm/643/12900 arXiv:1308.3743 (cit. on p. 5).

[Lei] T. Leinster, Basic bicategories. arXiv:math/9810017 (cit. on p. 4).

[LM16] J. A. Lind and C. Malkiewich, The transfer map of free loop spaces, 2016. arXiv:1604.03067 (cit on pp. $21,24,26)$.

[LM17] - The Morita equivalence between parametrized spectra and module spectra, 2017. arXiv:1702.07794 (cit. on p. 26). 
[MMSS01] M. A. Mandell, J. P. May, S. Schwede, and B. Shipley, Model categories of diagram spectra, Proc. London Math. Soc. (3) 82 (2001), no. 2, 441-512. doi:10.1112/S0024611501012692 (cit. on p. 5).

[MS06] J. P. May and J. Sigurdsson, Parametrized homotopy theory, Mathematical Surveys and Monographs, vol. 132, American Mathematical Society, Providence, RI, 2006. arXiv:math/0411656 (cit on pp. 4, 9, 12).

[Pon10] K. Ponto, Fixed point theory and trace for bicategories, Astérisque (2010), no. 333, xii+102. arXiv:0807.1471 (cit on pp. 1, 2, 3, 4, 7, 10, 18, 23, 25, 26).

[Pon16]_ Coincidence invariants and higher Reidemeister traces, J. Fixed Point Theory Appl. 18 (2016), no. 1, 147-165. doi:10.1007/s11784-015-0269-5 arXiv:1209.3710 (cit on pp. 2, 23, 25).

[PS12] K. Ponto and M. Shulman, Duality and traces for indexed monoidal categories, Theory Appl. Categ. 26 (2012), No. 23, 582-659. arXiv:1211.1555 (cit on pp. 17, 18).

[PS13] Shadows and traces in bicategories, J. Homotopy Relat. Struct. 8 (2013), no. 2, 151-200. doi:10.1007/s40062-012-0017-0 arXiv:0910.1306 (cit on pp. 3, 7, 11, 12, 14).

[PS14] _ The multiplicativity of fixed point invariants, Algebr. Geom. Topol. 14 (2014), no. 3, 1275-1306. doi:10.2140/agt.2014.14.1275 arXiv:1203.0950 (cit. on p. 11).

[Sch98] C. Schlichtkrull, The transfer map in topological Hochschild homology, J. Pure Appl. Algebra 133 (1998), no. 3, 289-316. doi:10.1016/S0022-4049(97)00117-5 (cit. on p. 23).

[Sch06] Transfer maps and the cyclotomic trace, Math. Ann. 336 (2006), no. 1, 191-238. doi:10.1007/s00208-006-0783-2 (cit. on p. 23).

[SS03] S. Schwede and B. Shipley, Equivalences of monoidal model categories, Algebr. Geom. Topol. 3 (2003), 287-334. doi:10.2140/agt.2003.3.287 (cit on pp. 6, 17).

[Seg74] G. Segal, Categories and cohomology theories, Topology 13 (1974), 293-312. doi:10.1016/0040-9383(74)90022-6 (cit. on p. 35).

[Shu08] M. Shulman, Framed bicategories and monoidal fibrations, Theory Appl. Categ. 20 (2008), No. 18, 650-738. arXiv:0706.1286 (cit. on p. 18).

[Sta65] J. Stallings, Centerless groups-an algebraic formulation of Gottlieb's theorem, Topology 4 (1965), 129-134. doi:10.1016/0040-9383(65)90060-1 (cit. on p. 10).

[Wal85] F. Waldhausen, Algebraic K-theory of spaces, Algebraic and geometric topology (New Brunswick, N.J., 1983), Lecture Notes in Math., vol. 1126, Springer, Berlin, 1985, pp. 318419. doi:10.1007/BFb0074449 (cit. on p. 2).

[Wec41] F. Wecken, Fixpunktklassen. I, Math. Ann. 117 (1941), 659-671. doi:10.1007/BF01450034 (cit. on p. 2).

Department of Mathematics, VAnderbilt University, 1326 Stevenson Center, Nashville, TENNESSEE, 37240

Department of Mathematics, University of Kentucky, 719 Patterson Office Tower, LeXINGTON, KENTUCKY, 40506 